\title{
An Introduction to ANFIS Based Channel Equalizers for Mobile Cellular Channels
}

\author{
K. C. Raveendranathan \\ Government Engineering College Bartonhill Vanchiyoor P.O. \\ India
}

\section{Introduction}

The purpose of a communication system is to transfer information between two separate points over some medium in the presence of disturbances or distortions such as noise and dispersion. This distortion is manifested in the time domain as pulse dispersion and is labeled as Inter-Symbol Interference (ISI). As data rates increase in modern digital communication systems, ISI becomes an inevitable consequence of the dispersive nature of band-limited propagation channels. The receiver must include an equalizer to mitigate the effects of ISI. The function of the equalizer is to combat the ISI and to utilize the available spectrum most efficiently.

Equalizers are cascaded to almost all kinds of channels, right from telephone lines to radio and optical fiber channels, to make the channel performance optimal. Ideally, an equalizer, when cascaded to the end of a channel, will make it behave like an ideal channel, the one which will not distort the signals in any manner. In the case of mobile cellular channels, which are generally considered to be Non-Linear and Time Variant (NLTV), the design of equalizers is not a trivial problem. Moreover, the above said channel has certain uncertainties in its behaviour, which need to be tackled in the equalizer design. The Co-Channel Interference (CCI) due to frequency reuse and Adjacent Channel Interference (ACI) due to spectral leakage, both contribute to the reduction in overall Signal-to-Interference-NoiseRatio (SINR) in mobile cellular channels.

In applications in which the Channel Impulse Response (CIR) is unknown and no training sequence is available, the equalizer must be computed/ updated blindly from the received signal and knowledge of the statistics of the data source alone. A common approach in continuous transmission systems is to blindly update a Linear Equalizer (LE) using the Constant Modulus Algorithm (CMA), and then switch to a Decision Directed (DD) mode when the Symbol Error Rate (SER) is low enough (Widro, 1973).

\section{Fading characteristics of mobile channels}

In mobile cellular radio transmission between a base station and a mobile telephone, the signal transmitted from the base station to the mobile receiver is usually reflected from surrounding buildings, hills, and other obstructions. As a consequence, we observe multiple propagation paths arriving at the receiver at different delays. Hence the received signal has characteristics similar to those for ionospheric propagation. The same is true for 
transmission from the mobile telephone to the base station. Moreover, the speed that the mobile (automobile, train, etc.) is traveling results in frequency offsets, called Doppler shifts, of the various frequency components of the signal (Proakis \& Salehi, 2002). As the intervening medium changes its characteristics with respect to time, the mobile radio channel is time varying.

An important requirement for assessing technology for mobile radio applications is to have an accurate description of the wireless channel. Channel models are heavily dependent upon the radio architecture. For example, in the first generation systems, a super-cell or "single-stick" architecture is used where the Base Transmitting Station (BTS) and the subscriber station are in Line-Of-Sight (LOS) condition and the system uses a single cell with no co-channel interference. For the second generation systems, a scalable multi-cell architecture with Non-Line-Of-Sight (NLOS) conditions becomes necessary.

The mobile cellular channel can be modeled as one of the following:

- The Free Space model.

- $\quad$ Tapped Delay Line (TDL) Channel model.

- $\quad$ Rayleigh Fading Channel model.

- Ricean Fading Channel model.

- $\quad$ The Hata-Okumura model.

- Cost 231 Walfish-Ikegami (W-I) model.

- Modified Stanford University Interim (SUI) Channel model.

- The FCC model.

- $\quad$ The ITU-R model.

The free space model is the simplest model, but it does not model the terrestrial environment reliably. A general model for a time-variant multipath channel is a TDL structure. It consists of a tapped delay line with uniformly spaced taps. The tap spacing between adjacent taps is $1 / \mathrm{W}$, where $W$ is the bandwidth of the signal transmitted through the channel. The tap coefficients, denoted as $\left\{c_{n}(t) \equiv a_{n}(t)\right.$ ejqn(t) $\}$ are usually modeled as complex valued, Gaussian random processes which are mutually uncorrelated (Proakis \& Salehi, 2002). The most widely used path loss model for signal strength prediction and simulation in macrocellular environments is the Hata-Okumura model (Okumura et.al., 1968, Hata, 1980). This model is valid for the 500-1500 $\mathrm{MHz}$ frequency range, receiver distances greater than $1 \mathrm{~km}$ from the base station, and base station antenna heights greater than $30 \mathrm{~m}$. It is shown that the Cost 231 Walfish-Ikegami (WI) model matches extensive experimental data for flat suburban and urban areas with uniform building height (Smith \& Dalley, 2000). It has also been found that the suburban path loss model for the Category C (flat terrain, light tree density) is in a good agreement with the Cost $231 \mathrm{~W}-\mathrm{I}$ model for suburban areas, providing continuity between the two proposed models. The Modified Stanford University Interim channel model can be used for simulations, design, and development and testing of technologies suitable for fixed broadband wireless applications. The FCC propagation model has three basic elements that affect the predicted field strength at the receiver, viz. Line-Of-Sight (LOS) mode, using basic free-space path loss; Non-Line-Of-Sight (NLOS) mode, using multiple wedge diffraction; and Partial first Fresnel zone obstruction losses applicable to either mode. The ITU-R model has the following salient features:

- It takes into account various physical phenomena such as line-of-sight, diffraction, tropospheric scatter, surface ducting, elevated layer reflection and refraction and hydrometeor scatter. 
- It uses the Deygout method, for multiple diffraction.

- Path loss is calculated for clear line-of-sight, line-of-sight with sub-path obstruction and trans-horizon cases.

- In analyses, which include terrain information, the FCC or ITU-R models are recommended.

- In analyses, which do not include terrain information, the FCC or ITU-R models can be used, provided that the model for the terrain profiles can be justified.

The "two ray" model is recommended for simple analyses, in which the propagation conditions are clearly line-of sight. To keep it simple, we can choose one or two models that will be the most conclusive and will cover most common cases. We have used the Rayleigh Fading and Ricean Fading Channel models in our discussion.

\section{Modelling and simulation of mobile channels}

The modeling and simulation of mobile cellular channels have been successfully carried out by several researchers. Various interference patterns including Ricean/Rayleigh fading, co-channel and adjacent-channel interferences can be found in literatures. In this Chapter, we intend to model the mobile cellular channel used in an indoor environment, where the channel can be taken to be of slow fading type. The study is focused to consider the noise contributions from various sources, when they fall within the spectrum of the frequencies used in cellular telephony, and then to design an equalizer which will mitigate the noise present, due to CCI and ACI. When the channel over which data is sent is unknown, which is common, one must employ adaptive equalization. The Decision Feedback Equalizer (DFE) is one such adaptive equalizer. It is known that DFE generally outperforms Linear Equalizer (LE) for the same hardware complexity. Further, as it is indicated earlier, when the channel characteristics show Rayleigh/Ricean fading (due to the presence of multipath), Adjacent Channel Interference (ACI) and Co-Channel Interference (CCI), realization of equalizers based on neuro-fuzzy techniques seems to be most appropriate option for the mobile cellular channel.

Linear space-time equalization is shown to be effective in coping with the complicated propagation conditions for wireless broadband communication in an industrial, indoor environment. This is demonstrated by realistic simulations that use real channel sounder for modeling the influence of the radio channel. Industrial indoor environments like large factory halls show typically a complicated radio channel because of the presence of many reflecting objects. This results in wide delay spreads and a considerably changing channel for a moving mobile unit. There exist a number of options to overcome the difficulties of heavy multipath propagation.

The issues in the design of the neuro-fuzzy channel equalizer to null the effects of fading are investigated. One of the objectives of this article is, to establish the fact that, within an acceptable bound, the mobile cellular channel is Linear Time Variant (LTV). Another major objective of this article is to investigate the suitability of neuro-fuzzy models as applicable to the analysis and design of mobile cellular channel equalizers. Three solutions to the channel equalizer problem are investigated in this article. First, a type-2 Fuzzy Adaptive Filter (FAF) for the above purpose is considered. Simulations show that it performs better than a type-1 FAF or Neural Network Classifier (NNC) equalizer. Then the use of Adaptive Network Based Fuzzy Inference System (ANFIS) based equalizer is investigated. Lastly, a Compensatory Neuro-Fuzzy Filter (CNFF) for channel equalization is considered (Lin \& Ho, 2004). 
Subsequently, an attempt is made to bring the various equalizer realizations in the study under the generic framework of radial basis function (RBF) neural network. Further to this, a novel modular approach for the simulation and design of equalizers for Non-Linear TimeVariant (NLTV) channels is aimed. A suitable model for Ultra-Wide Band (UWB) channel and its equalization is the last goal. It may be noted that the mobile channels are considered to be Linear, and Time Variant (LTV), as a limiting case. However, when the transmitter stages are driven to their non-linear regions, the channel need to be modeled as Non-Linear (to account for the non-linearities thus introduced to the transmitted signal) and TimeVariant (NLTV). The modular approach in combating ISI is to cascade an adaptive nonlinear preprocessor filter and linear adaptive equalizer, which simplifies the equalizer design. It is also shown that the ANFIS model can be successfully adapted to equalization of UWB channels.

\section{Channel equalization of mobile channels}

Channel equalizers are used in digital communication receivers to mitigate the effects of Inter-Symbol Interference (ISI) and inter user interference in the form of Co-Channel Interference (CCI) and adjacent channel interference (ACI) in the presence of Additive White Gaussian Noise (AWGN). Linear equalizers based on adaptive filtering techniques have long been used for this application. Recently, use of nonlinear signal processing techniques like Artificial Neural Networks (ANN) and Radial Basis Functions (RBF) has shown encouraging results in this application. In order to facilitate digital data communication with bandwidth efficiency, we need to use adaptive equalizers. There have been many solutions to the problem of active noise cancellation in mobile indoor channels. Several investigators have studied various active noise cancellers in detail. The main objectives of this article are modeling of a mobile broadband communication channel and designing of a Neuro-Fuzzy Adaptive Equalizer for it. In digital transmission, the problem of Inter-Symbol Interference (ISI) is most often mitigated by the receiving-end equalization. The two important issues in the design and implementation of equalizers are its complexity and its training.

The advancements in Digital Signal Processing (DSP) provide scope for very large scale implementation of many complex algorithms in a lucid manner. The programming capability of the Digital Signal Processors (DSP) makes them very suitable for fast realization. This is definitely an advantage while developing a new system based on an unproven technology, as modifying the design is a matter of re-programming the DSP chip. Thus the product development cycle gets simplified. And, modifications can be incorporated quite easily. Due to the reasons mentioned above, non-linear equalizers are being investigated by many researchers. They include Artificial Neural Networks (ANN), Radial Basis Functions (RBF), recurrent networks and neuro-fuzzy systems.

\subsection{Nomenclature of channel equalizers}

Recent advances in nonlinear signal processing techniques have provided a rich variety of nonlinear equalizers. Some of the equalizers developed with these processing techniques are based on Volterra filters, ANN, perceptrons, Multi Layer Perceptrons (MLP), RBF networks, fuzzy filters and fuzzy basis functions. All of these nonlinear equalizers, during their training period, optimize some form of a cost function like the mean square error (MSE) or probability of error and have the capability of providing the optimum Bayesian equalizer performance in terms of BER. 


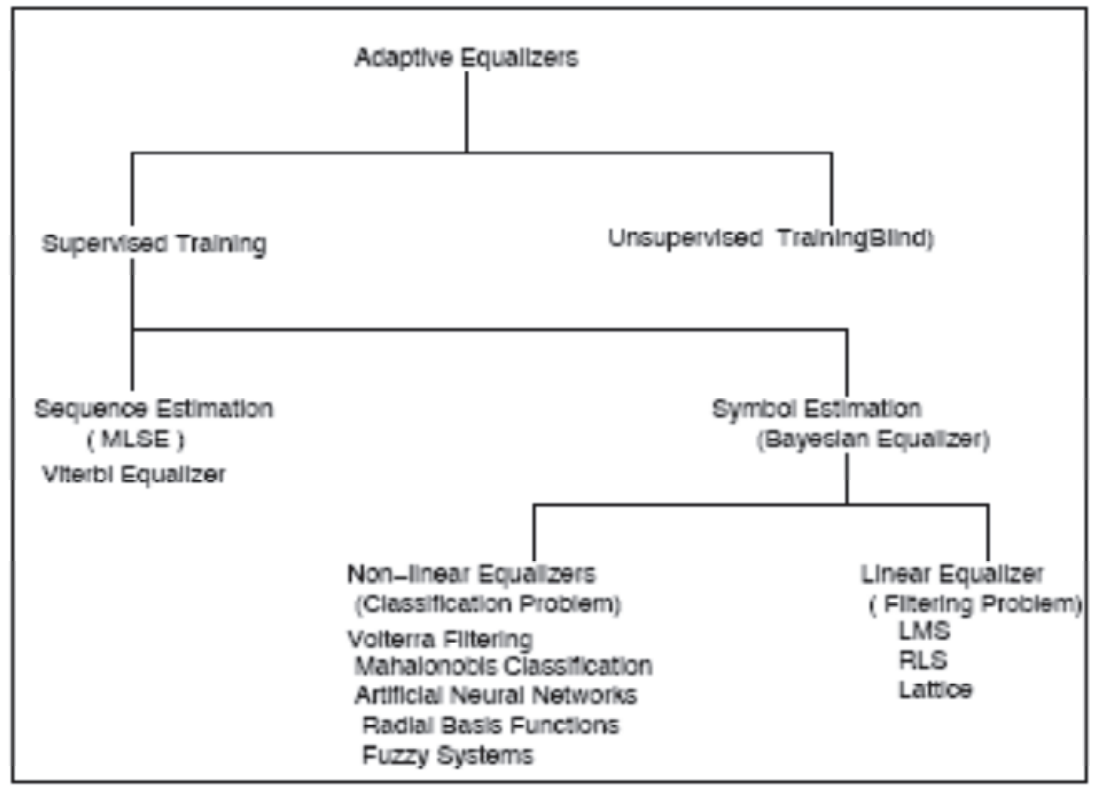

Fig. 1. Classification of Equalizers.

The nonlinear equalizers treat equalization as a pattern classification process where the equalizer attempts to classify the input vector into a number of transmitted symbols. The fuzzy equalizers investigated in this Chapter fall into this category. The Figure 1 shows a typical classification of equalizers. In general the family of adaptive equalizers can be classified as supervised equalizers and unsupervised equalizers.

\section{Neuro fuzzy adaptive equalizers for cellular channels}

Fuzzy logic and neural networks (with genetic algorithms) are complementary technologies in the design of intelligent systems (Wang \& Mendel, 1993). Fuzzy logic is based on the way the brain deals with inexact information, while neural networks are modeled after the physical architecture of the brain. Fuzzy systems and neural networks are both numerical model-free estimators and dynamic systems. They share the common ability to improve the intelligence of systems working in an uncertain, imprecise, and noisy environment. Neural networks provide fuzzy systems with learning abilities, and fuzzy systems provide neural networks with a structured framework with high-level fuzzy IF-THEN rule thinking and reasoning. The fuzzy adaptive filter is constructed from a set of fuzzy IF-THEN rules that change adaptively to minimize some criterion function as new information becomes available. The concept can be generalized to include complex parameters and complex signals. The fuzzy filter as adaptive equalizer is applied to Quadrature Amplitude Modulation (QAM) digital communication with linear complex channel characteristics.

The fuzzy adaptive filter has drawn a great deal of attention because of its universal approximation ability in nonlinear problems. These fuzzy rules come either from human experts or by matching input-output pairs through an adaptation procedure. Some 
application examples of the fuzzy filter to signal processing include classification and signal prediction, communications channel equalization, and nonlinear systems modeling and identification. Most fuzzy filters available to us, are real-valued and are suitable for signal processing in real multidimensional space. In some applications, however, signals are complex valued and processing is done in complex multidimensional space, An example is the equalization of digital communication channels with complex signaling schemes such as QAM. For complex signal processing problems, many existing fuzzy filters cannot directly be applied. It may be emphasized that the cellular, mobile communication channel is essentially a non-linear, time varying, fading channel. Therefore, the design of the equalizer is, it follows that, primarily a non-linear problem. Thus it becomes imperative that we can design appropriate equalizers for wireless fading channels by employing non-linear techniques. Several researchers have come up varying solutions to this daunting problem. In fact, the adaptive equalization is a highly challenging problem, a fact which is evident from the large research literature available and forthcoming.

\subsection{The type-2 fuzzy adaptive filter}

The type-2 fuzzy adaptive filter for channel equalization is obtained by generalizing the unnormalized output type-1 Takagi-Sugeno-Kang (TSK) fuzzy logic system to a type-2 TSK fuzzy logic system (Liang \& Mendel, 2000). For equalization, the antecedents of type-1 TSK FLS are generalized to type-2 fuzzy sets, whereas the consequent is unchanged (i.e., it is a crisp number). In a type-2 FAF with a rule base of $M$ rules, where each rule has $p$ antecedents, the $i^{\text {th }}$ rule $R^{i}$ is denoted as:

$$
R^{i}: \text { IF } x_{1}{ }^{i} \text { is } F_{1}{ }^{i} \text { and } x_{2}{ }^{i} \text { is } F_{2}{ }^{i} \text { and } \ldots x_{p}{ }^{i} \text { is } F_{p}{ }^{i} \text { THEN } y^{i}=c_{0}{ }^{i}+c_{1}{ }^{i} x_{1}{ }^{i}+c_{2}{ }^{i} x_{2} i+\ldots+c_{p}{ }^{i} x_{p}{ }^{i}
$$

where $i=1,2, \ldots, M ; c_{i}^{j}(j=0,1, \ldots, p)$ are the consequent parameters that are crisp numbers; $y^{i}$ is an output from the IF-THEN rule, which is a crisp number and the $F_{i} k(k=1,2, \ldots, p)$ are type-2 fuzzy sets.

\section{Performance of various equalizers}

We will now examine the performance of typical equalizers employed in wireless, mobile, cellular channels. The block diagram of a typical digital communication system is shown in Figure 2.. It clearly illustrates where the equalizer firs in as a part of the receiver sub system.

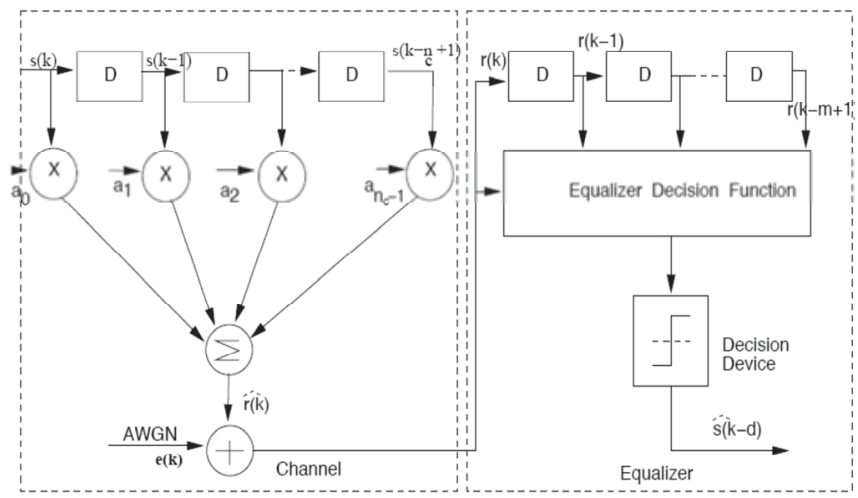

Fig. 2. The Block Diagram of a Typical Digital Communication System. 
Typical performance of decision feedback equalizer (DFE), which is a nonlinear system, is shown in Figure 3, below:

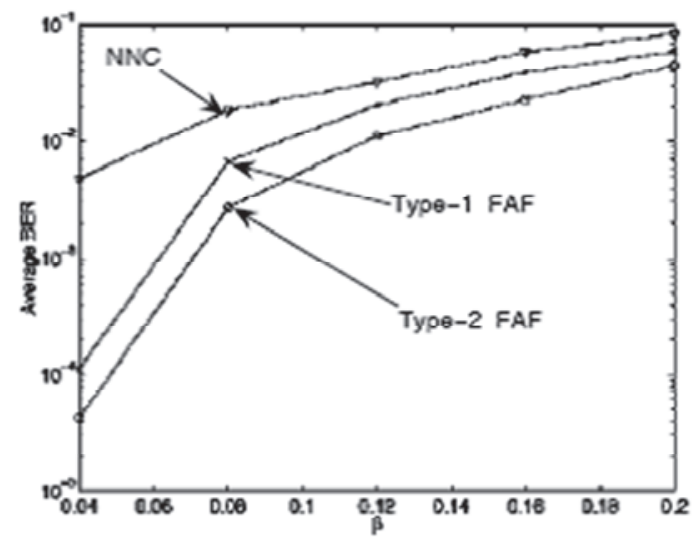

(a)

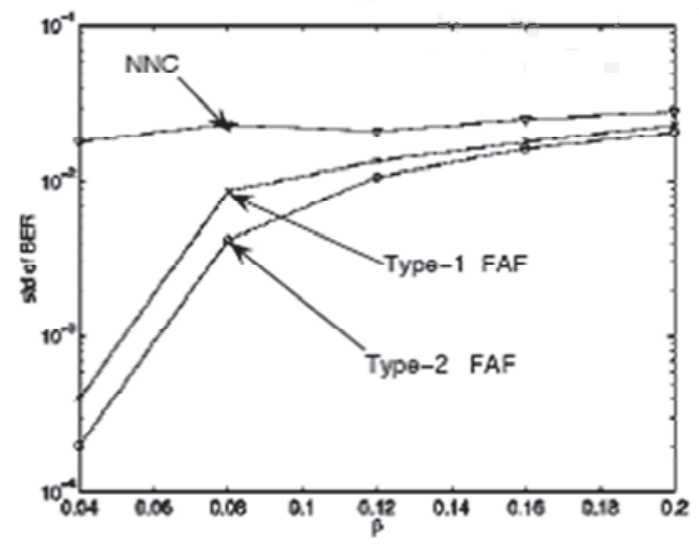

(b)

Fig. 3. Performance DFE based on Fuzzy Adaptive Filters (FAF). (a) Mean BER versus std of AWGN. (b) std of BER versus std of AWGN.

We have the following inferences based on the performance curves of type- 1 and type-2 FAF (denoted as FAF-I and FAF-II) and neural network classifiers (NNC):

- Both the type-2 FAF TE and DFE perform better than either a type-1 FAF or a Nearest Neighbour Classifier (NNC).

- Since no tuning procedure is used in the design of either type-2 FAF based equalizer, real-time information processing is guaranteed.

- It will be of great advantage to develop a FAF based Blind Channel Equalizer.

\section{The ANFIS based channel equalizer}

In this section, we discuss in detail, the Adaptive Network Based Fuzzy Inference System (ANFIS) and associated equalizer models for mobile cellular and UWB channels. The theory 
of ANFIS was introduced by J.S.R. Jang in his seminal paper in (Jang, 1993). It may be noted that the equalization of wireless mobile channels is a non-linear problem, so that a nonlinear solution, such as ANFIS, is more appropriate. One has to design the fuzzy if-then else rules based on the channel characteristics; namely variances of signal, noise, co-channel (CCI) and adjacent channel interferences (ACI) as well as the transmitted signal (input)received signal (output) mapping. The equalizer is a non-linear system that effectively undoes the aberrations done to the transmitted signal by the channel due to the noise and co-channel and adjacent channel interferences. Now, the modeling a non-linear system is fairly complex so that conventional methods of system identification techniques cannot be applied to find the inverse system. One possible experimental method to develop a model for indoor wireless channel (viz., the channel impulse response, CIR) is to carry out expensive channel sounding (for example, one could use the RUSK Channel sounder from RF Sub Systems, GmBH, which would cost over a hundred thousand dollars). In this article, we attempt to supplant the expensive channel sounding technique for mobile wireless channel (that too, not restricted to the indoor case) by suitable simulation techniques.

\subsection{Introduction to ANFIS}

Functionally, there are almost no constraints on the node functions of an adaptive network except piecewise differentiability. Structurally, the only limitation of network configuration is that it should be of feedforward type. Due to these minimal restrictions, the adaptive network's applications are immediate and immense in various areas. For simplicity, we assume the fuzzy inference system under consideration has two inputs $x$ and $y$ and one output $z$ (Jang, 1993). Suppose that the rule base contains two fuzzy if-then rules of Takagi and Sugeno's type:

Rule1 : If $\mathrm{x}$ is $\mathrm{A} 1$ and $\mathrm{y}$ is $\mathrm{B} 1$, then $\mathrm{f} 1=\mathrm{p} 1 \mathrm{x}+\mathrm{q} 1 \mathrm{y}+\mathrm{r} 1$.

Rule2: If $x$ is $A 2$ and $y$ is $B 2$, then $f 2=p 2 x+q 2 y+r 2$.

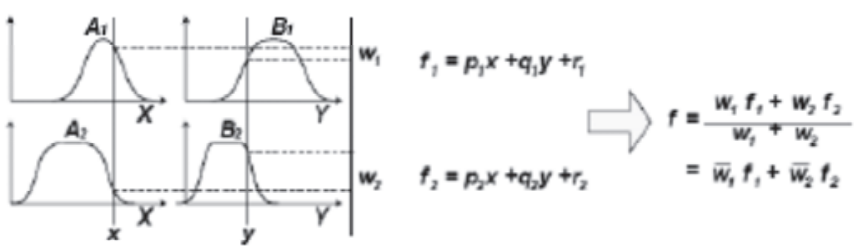

(a)

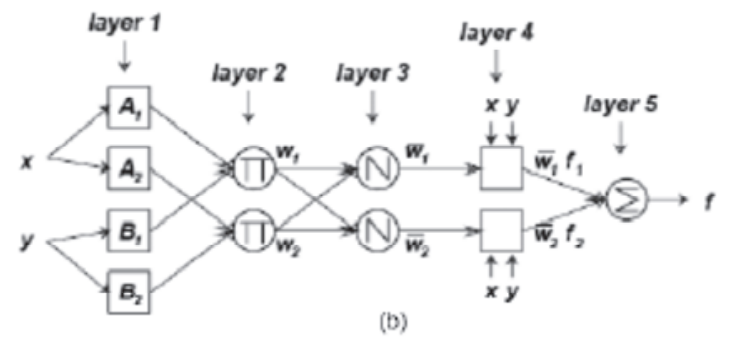

Fig. 4. The Takagi-Sugeno-Kang (TSK) Model of Fuzzy Reasoning. (a) Type-3 Fuzzy Reasoning. (b) Equivalent ANFIS (Type-3 ANFIS). 
The type-3 fuzzy reasoning is illustrated in Figure 4(a) and the corresponding equivalent ANFIS architecture (type-3 ANFIS) is shown in Figure 4(b).

\subsection{Mobile cellular channel equalization based on ANFIS}

One can observe that wireless channel can be modeled as non-linear time-variant (NLTV) when the duration of observation window is fairly long or as non-linear time invariant (NLTI) when the duration of observation window is short. This fact is established by simulation, as it is a hard problem to obtain a rigorous mathematical proof. Conventional channel models available in recent literature were studied to arrive at a suitable paradigm for the wireless channel, consisting of the different variables and parameters. This also enabled us to understand the inadequacies of existing mathematical models for wireless channels. The fuzzy if-then rules are generated by the inverse system based on ANFIS (to the channel), which effectively acts as an adaptive equalizer at the receiver side. The ANFIS automatically generate the rule base from a set of input-output data vectors. This is achieved by minimizing the error between actual input signal (at the transmitter of the wireless system) and the estimate of the input (at the receiver). In the simulation, we assume that the external input to the ANFIS equalizer is the output of the channel, which is the sum of the desired channel output plus the weighted sum of the co-channel outputs and the Gaussian noise, which is assumed to be AWGN, with zero mean and standard deviation upto 0.8. In the ensuing sections, we use the following definitions for Signal-toNoise Ratio (SNR), Signal-to-Interference Ratio (SIR) and Signal-to-Interference Noise Ratio (SINR) (Liang \& Mendel 2000):

$$
\begin{gathered}
\text { SNR }=10 \log _{10}\left\{\sigma_{\mathrm{s}}{ }^{2} / \sigma_{\mathrm{n}}{ }^{2}\right\} \\
\mathrm{SIR}=10 \log _{10}\left\{\sigma_{\mathrm{s}}{ }^{2} / \sigma_{\mathrm{i}}{ }^{2}\right\} \\
\mathrm{SINR}=10 \log _{10}\left\{\sigma_{\mathrm{s}}{ }^{2} /\left(\sigma_{\mathrm{i}}{ }^{2}+\sigma_{\mathrm{n}}{ }^{2}\right)\right\}
\end{gathered}
$$

where $\sigma_{s}^{2}, \sigma_{n}^{2}$, and $\sigma_{i}^{2}$ are the variances of the signal, AWG noise, and the co-channel and adjacent channel interferences (put together) signal respectively.

\begin{tabular}{|c|c|c|c|}
\hline Type & Nodes & Linear/Nonlinear Parameters & Fuzzy Rules \\
\hline ANFIS-17 & 32 & $14 / 14$ & 7 \\
\hline ANFIS-115 & 64 & $30 / 30$ & 15 \\
\hline ANFIS-125 & 104 & $50 / 50$ & 25 \\
\hline ANFIS-25 & 75 & $75 / 20$ & 25 \\
\hline ANFIS-27 & 131 & $147 / 28$ & 49 \\
\hline ANFIS-35 & 286 & $500 / 30$ & 125 \\
\hline ANFIS-37 & 734 & $1372 / 42$ & 343 \\
\hline
\end{tabular}

Table 1. Simulation Parameters for Various ANFIS Based Channel Equalizers

The output of the equalizer is given to a limiter to clip the output levels to limiting values of +1 or -1 . The different parameters of the various simulation setups are as tabulated in Table 1. The structure of ANFIS-27 is given in Figure 5. The library function, anfis, available in the Fuzzy Logic Toolbox of MATLAB $®$ R2010b is used extensively in all simulations. 


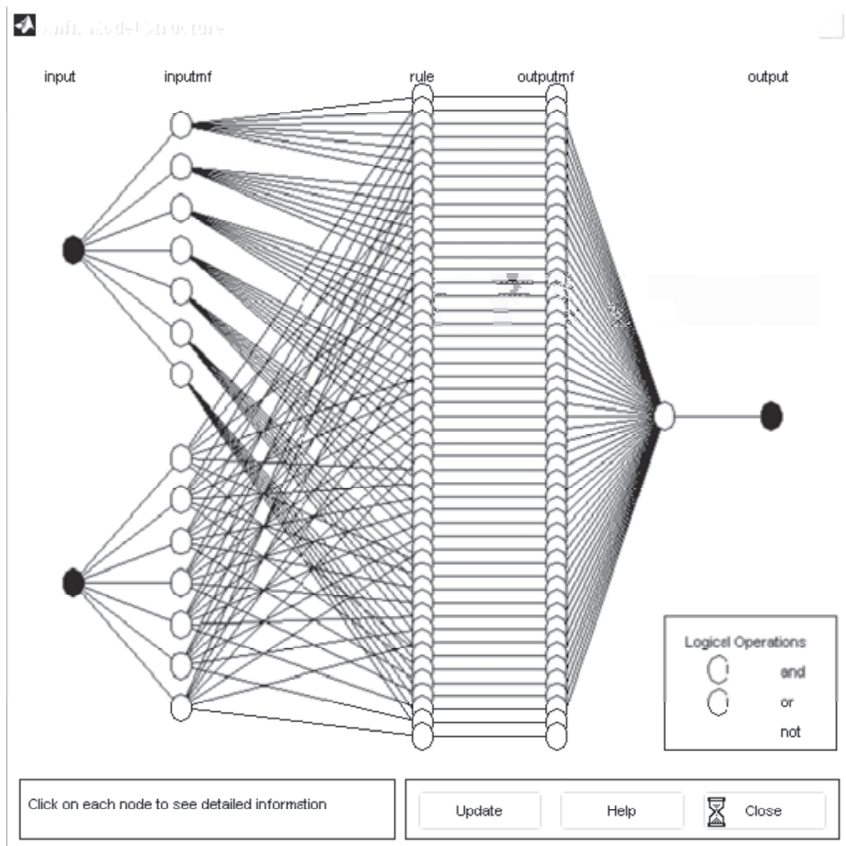

Fig. 5. The Structure of ANFIS-27 Generated Using MATLAB Fuzzy Logic Toolbox.

Note that ANFIS-27 based equalizer has two inputs from multipath components, seven (7) fuzzy rules for each input and one output that feed the receiver subsystem.

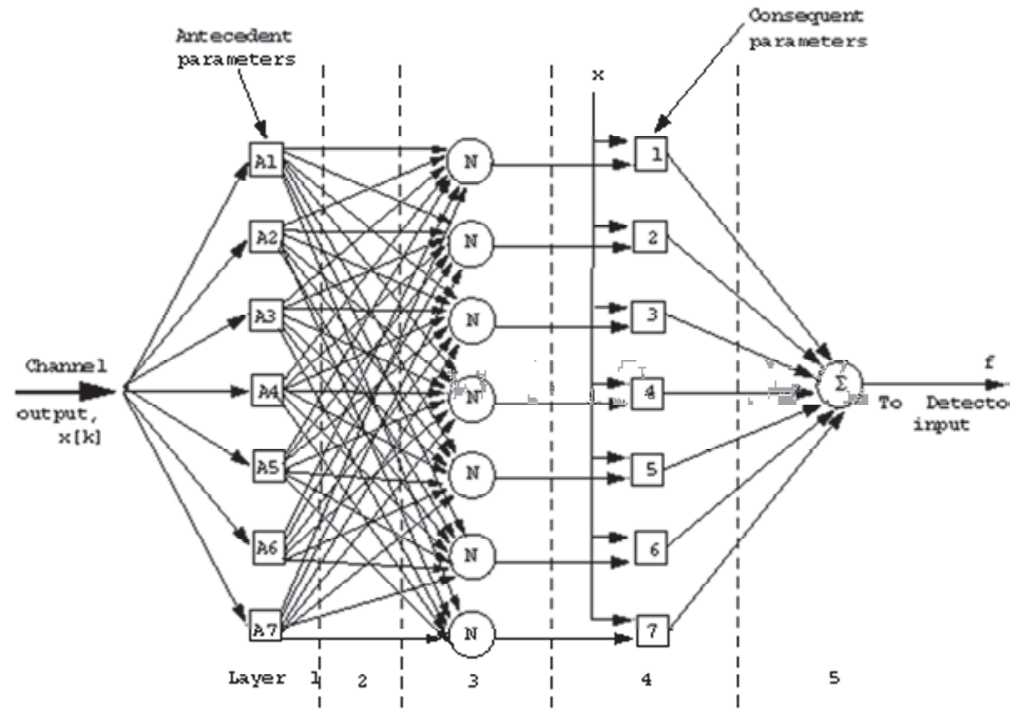

Fig. 6. The Equivalent ANFIS Architecture for Channel Equalizer. 
The Figure 6 shows the architecture of the proposed ANFIS based channel equalizer, for 7 fuzzy rules. The wireless channel modeling based on artificial neural networks is capable of depicting the input-output mapping existing in the equalizer system and it does provide us with an exact picture of the variables and parameters defining the system. Moreover, neural network based models do have the learning capability. The fuzzy models, on the other hand, do not possess the learning capability. Therefore, fusing together these two, we can have a model which is capable of both depicting the dynamics of the system in terms of the variables and parameters and is having the self-learning capability. The adaptability of the equalizer under purview is achieved by the learning aspect of neural network. The fuzzy reasoning (especially the TSK model used in ANFIS) maps the input to the output. We follow a first-order ANFIS with the antecedent parameters being the standard deviations of the received signal, CCI and ACI interferences (put together), and the AWGN $\left(\sigma_{s}, \sigma_{i}\right.$, and $\sigma_{n}$, respectively), collectively represented as $A_{i}$. The only consequent parameter is the scaling factor of the signal $\left(\rho_{i}\right)$ at the output. The membership functions of $A_{i}, i=1,2, \ldots, 7$ are chosen to be Gaussian. Some of the rules in the fuzzy rule base can be stated as:

If $\sigma_{\mathrm{s}}$ is very low, and $\sigma_{\mathrm{i}}$ is very low, and $\sigma_{\mathrm{n}}$ is very low then $y=\rho_{1} \mathrm{~s}$.

If $\sigma_{\mathrm{s}}$ is low, and $\sigma_{\mathrm{i}}$ is very low, and $\sigma_{\mathrm{n}}$ is very low then $\mathrm{y}=\rho_{2} \mathrm{~s}$.

If $\sigma_{\mathrm{s}}$ is medium, and $\sigma_{\mathrm{i}}$ is very low, and $\sigma_{\mathrm{n}}$ is very low then $\mathrm{y}=\rho_{3} \mathrm{~s}$.

If $\sigma^{\wedge} s$ is medium, and $\sigma_{\mathrm{i}}$ is low, and $\sigma_{\mathrm{n}}$ is low then $\mathrm{y}=\rho_{4} \mathrm{~s}$.

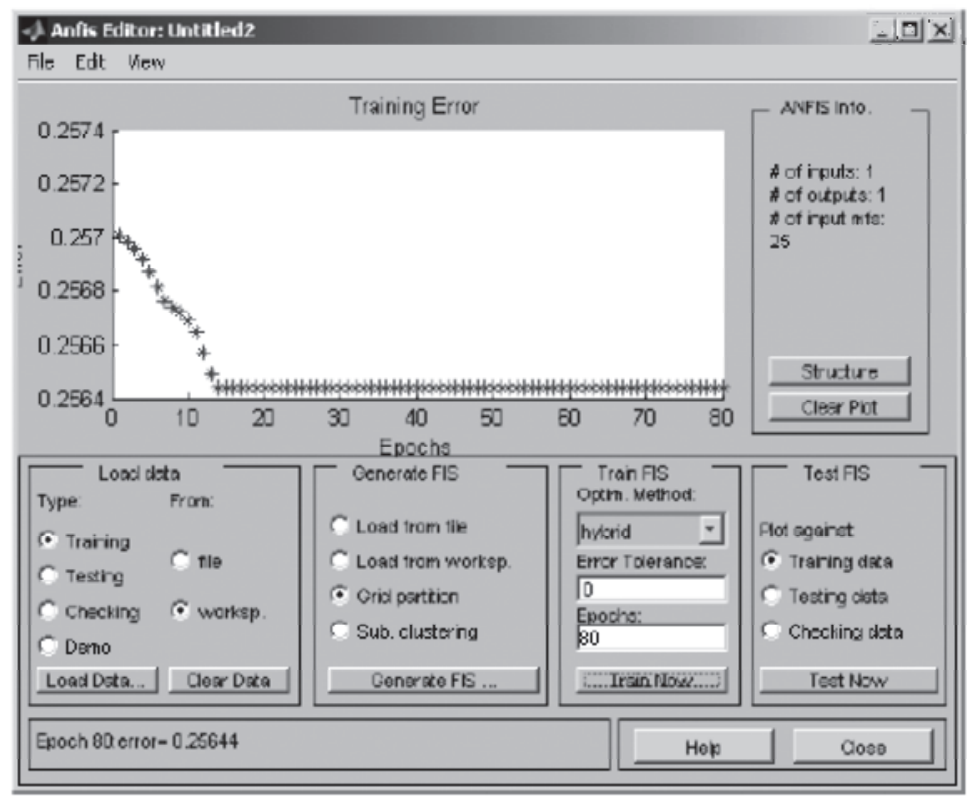

Fig. 7. The Error Plot of ANFIS-125 Training. 
The three input variables can assume any one of the 5 possible membership functions from the set, \{very low, low, medium, high, very high\}, leaving us with 125 possible combinations of rules. However, using fuzzy rule reduction techniques the total number of rules can be limited to 7 or 25. The steps in the algorithm for simulation of the ANFIS-27 based equalizer are as given below:

1. The standard deviations of CCI and AWGN are logarithmically varied from 0.02 to 0.8 . This information is derived from literature.

2. The random binary input data (which represents the input to the channel from the transmitter) is generated and the corrupted data available at the outputs of the two multipaths due to CCI and AWGN is obtained.

3. Set the number of membership functions as 7, membership function type as "Gaussian" and the number of epochs to 80 .

4. Simulate the ANFIS (which implements the equalizer) and plot the results.

The error plot of the ANFIS-125 training is illustrated in Figure 7.

We have set the number of epochs as 80 in this case. As the ANFIS implementation in MATLAB do not lend itself to observe the updation of Antecedent and consequent parameters, while training is under progress, we can consider the training error as a reliable pointer to the step wise updation of the above parameters. The ANFIS- 125 consists of one input, one output, and 25 fuzzy rules for each membership functions.

\subsection{The results of simulations of ANFIS based equalizers}

The simulated output of the channel, which is the input to the ANFIS based channel equalizer, along with the training data is shown in Figure 8. The output of the channel (received signal), which is a non-linear combination of the signal, the co-channel signals, and the AWG noise, is a random waveform taking values around +1 and -1 , as seen from the simulated waveform. The MATLAB code to generate the plot is given below.
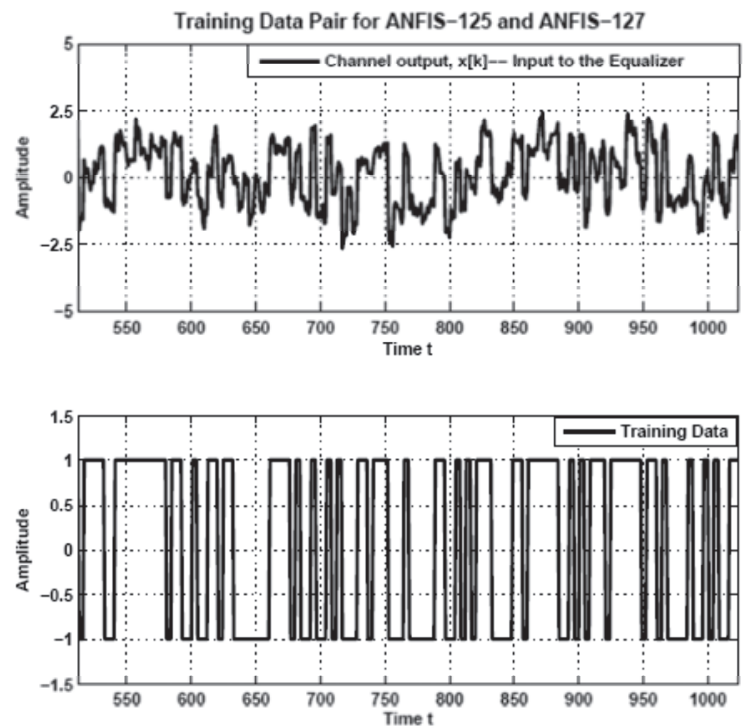

Fig. 8. The Training Data Pair for ANFIS-125 Equalizer Simulation. 
The equalized, output after thresholding, will be very much identical to the training data as shown in Figure 9.
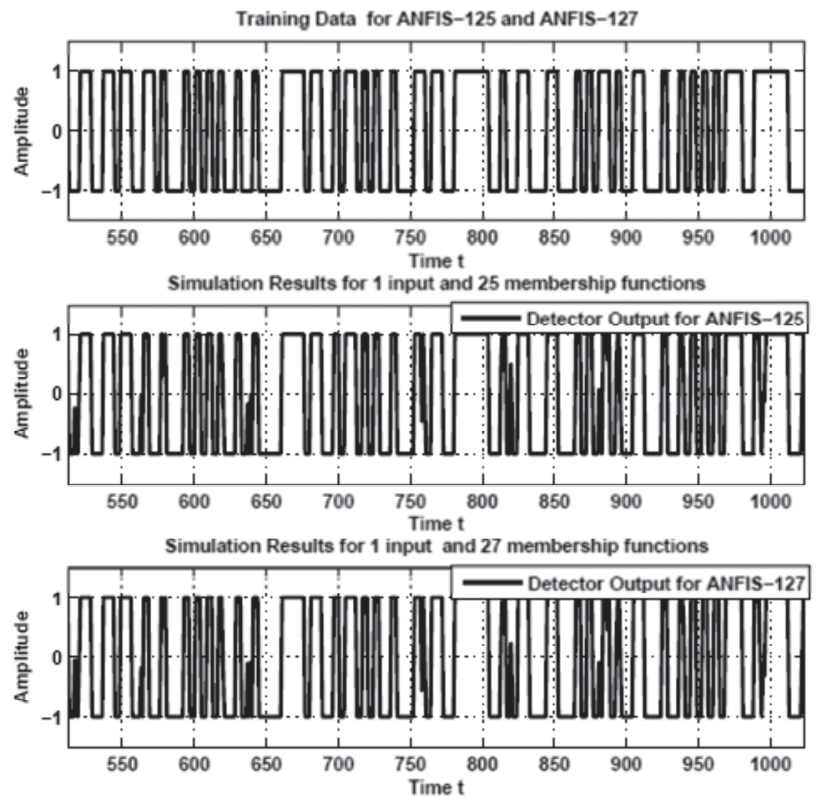

Fig. 9. Simulation Results for ANFIS-125 and ANFIS-127 based Equalizers.

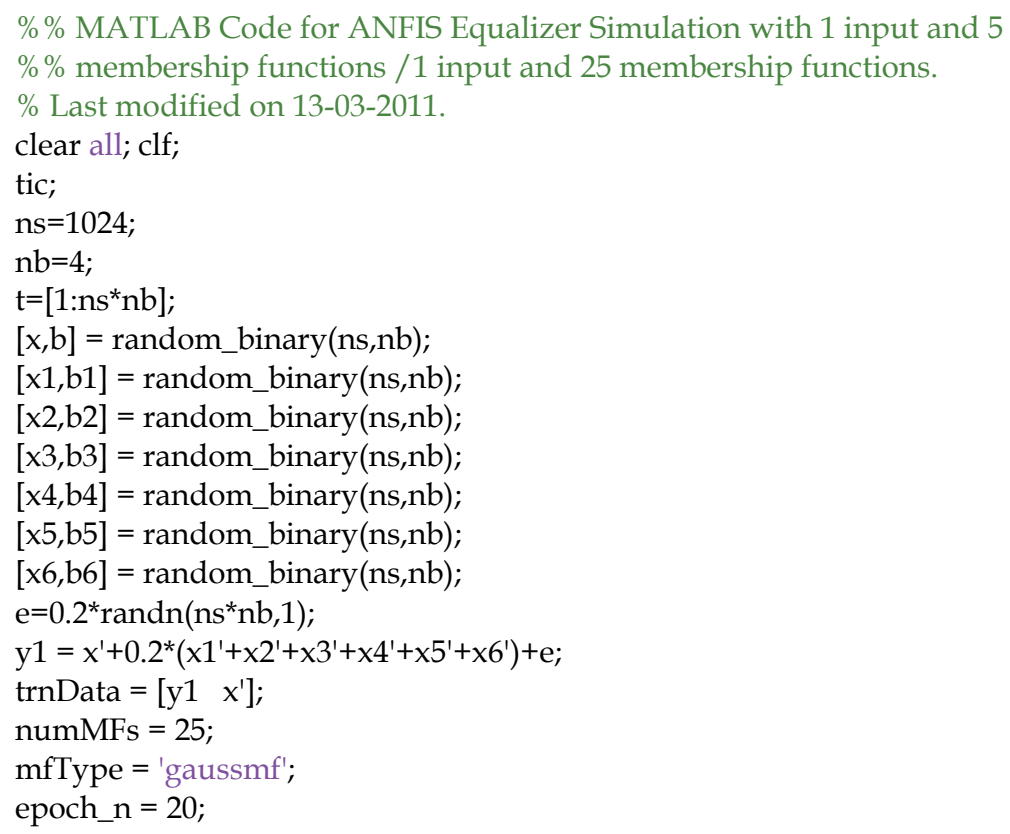


subplot(211), plot(t(512:1024),y1(512:1024),'k');

axis([512 1024 -5 5]); grid on;

xlabel('Time t');ylabel('Amplitude');

legend('Channel output, $x[\mathrm{k}]--$ Input to the Equalizer');

title('Training Data Pair for ANFIS-125 and ANFIS-127');

subplot(212), plot(t(512:1024),x(512:1024),''k');

axis([512 1024 -1.5 1.5]); grid on;

xlabel('Time t');ylabel('Amplitude');

legend('Training Data');

$\% \%$ ANFIS Equalizer Simulation with 1 input and 5 membership

$\% \%$ functions $/ 1$ input and 25 membership functions.

\% Last modified on 13-03-2011.

clear all;clf;

tic;

ns=1024;

$\mathrm{nb}=4$;

$\mathrm{t}=\left[1: \mathrm{ns}{ }^{*} \mathrm{nb}\right] ;$

$[\mathrm{x}, \mathrm{b}]=$ random_binary(ns,nb);

$[\mathrm{x} 1, \mathrm{~b} 1]=$ random_binary $(\mathrm{ns}, \mathrm{nb})$;

$[\mathrm{x} 2, \mathrm{~b} 2]=$ random_binary $(\mathrm{ns}, \mathrm{nb})$;

$[\mathrm{x} 3, \mathrm{~b} 3]=$ random_binary $(\mathrm{ns}, \mathrm{nb})$;

$[\mathrm{x} 4, \mathrm{~b} 4]=$ random_binary $(\mathrm{ns}, \mathrm{nb})$;

$[\mathrm{x} 5, \mathrm{~b} 5]=$ random_binary $(\mathrm{ns}, \mathrm{nb})$;

$[\mathrm{x} 6, \mathrm{~b} 6]=$ random_binary(ns,nb);

$\mathrm{e}=0.2^{*} \operatorname{randn}\left(\mathrm{ns}{ }^{*} \mathrm{nb}, 1\right)$;

$y 1=x^{\prime}+0.2^{*}\left(x 1^{\prime}+x 2^{\prime}+x 3^{\prime}+x 4^{\prime}+x 5^{\prime}+x 6^{\prime}\right)+e ;$

$\operatorname{trnData}=\left[\begin{array}{ll}\mathrm{y} 1 & \mathrm{x}^{\prime}\end{array}\right] ;$

numMFs $=25$;

mfType = 'gaussmf';

epoch_n $=20$;

in_fismat = genfis1(trnData,numMFs,mfType);

out_fismat $=$ anfis(trnData,in_fismat,20);

est_x125=evalfis(y1,out_fismat);

est_x125(est_x125<-0.6)=-1.0;

est_x125(est_x125>0.6)=1.0;

$\operatorname{trnData}=\left[\begin{array}{ll}\mathrm{y} 1 & \mathrm{x}^{\prime}\end{array}\right] ;$

numMFs = 27;

mfType = 'gaussmf';

epoch_n $=20$;

in_fismat = genfis1(trnData,numMFs, $m$ fType);

out_fismat = anfis(trnData,in_fismat,20);

est_x127=evalfis(y1,out_fismat); 


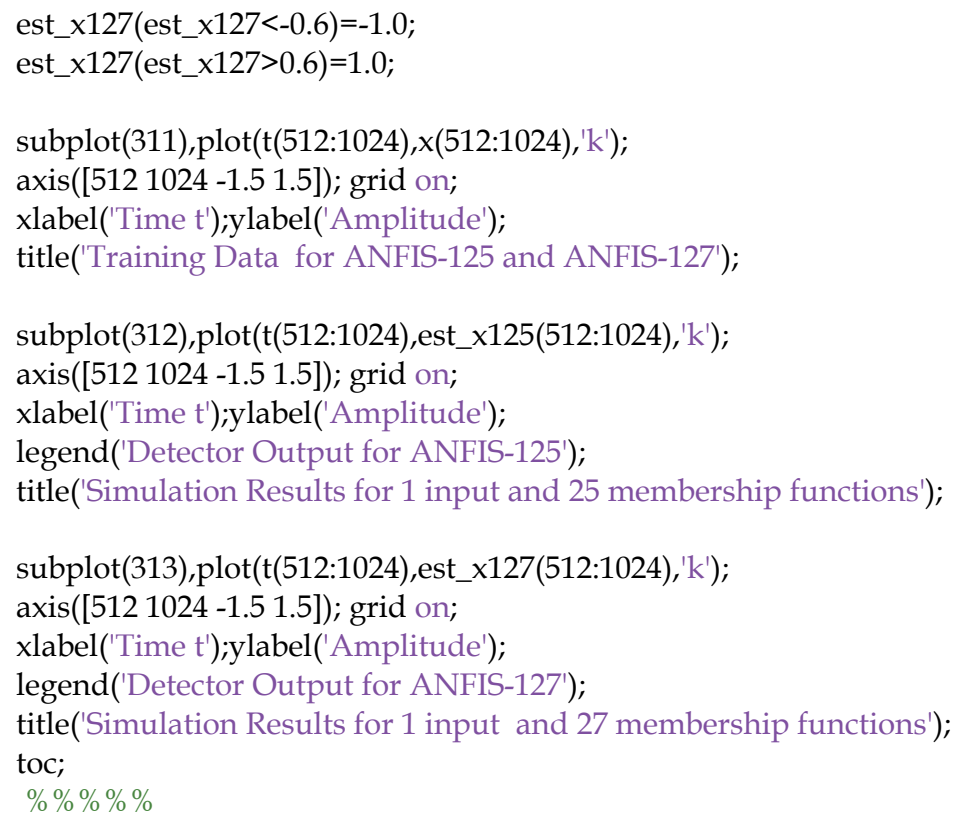

In one of the simulations, the standard deviation of CCI and AWGN are logarithmically varied between 0.02 and 0.8 and simulation is run on a total of 2048/4096 training data pairs. The results are shown in Figure 10. The MATLAB code to generate the same is appended below.

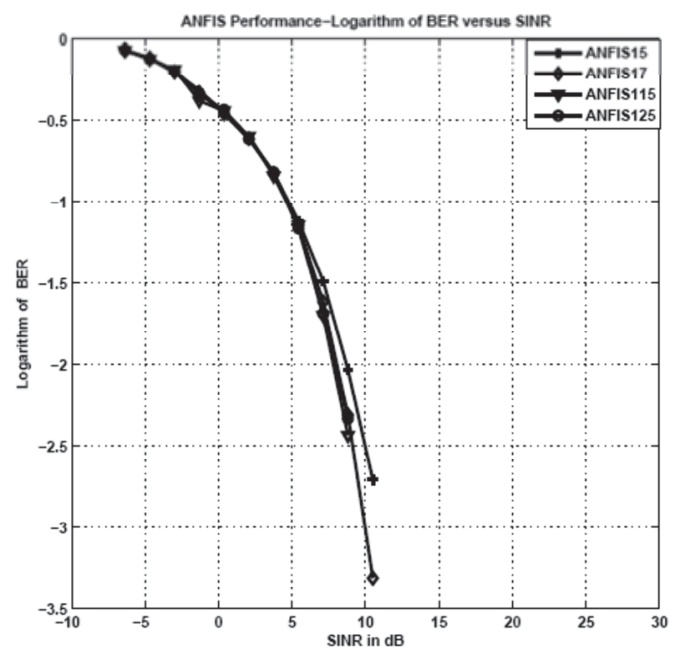

Fig. 10. Performance of ANFIS based Equalizer - Logarithm of BER at output versus SNR in dBs. 


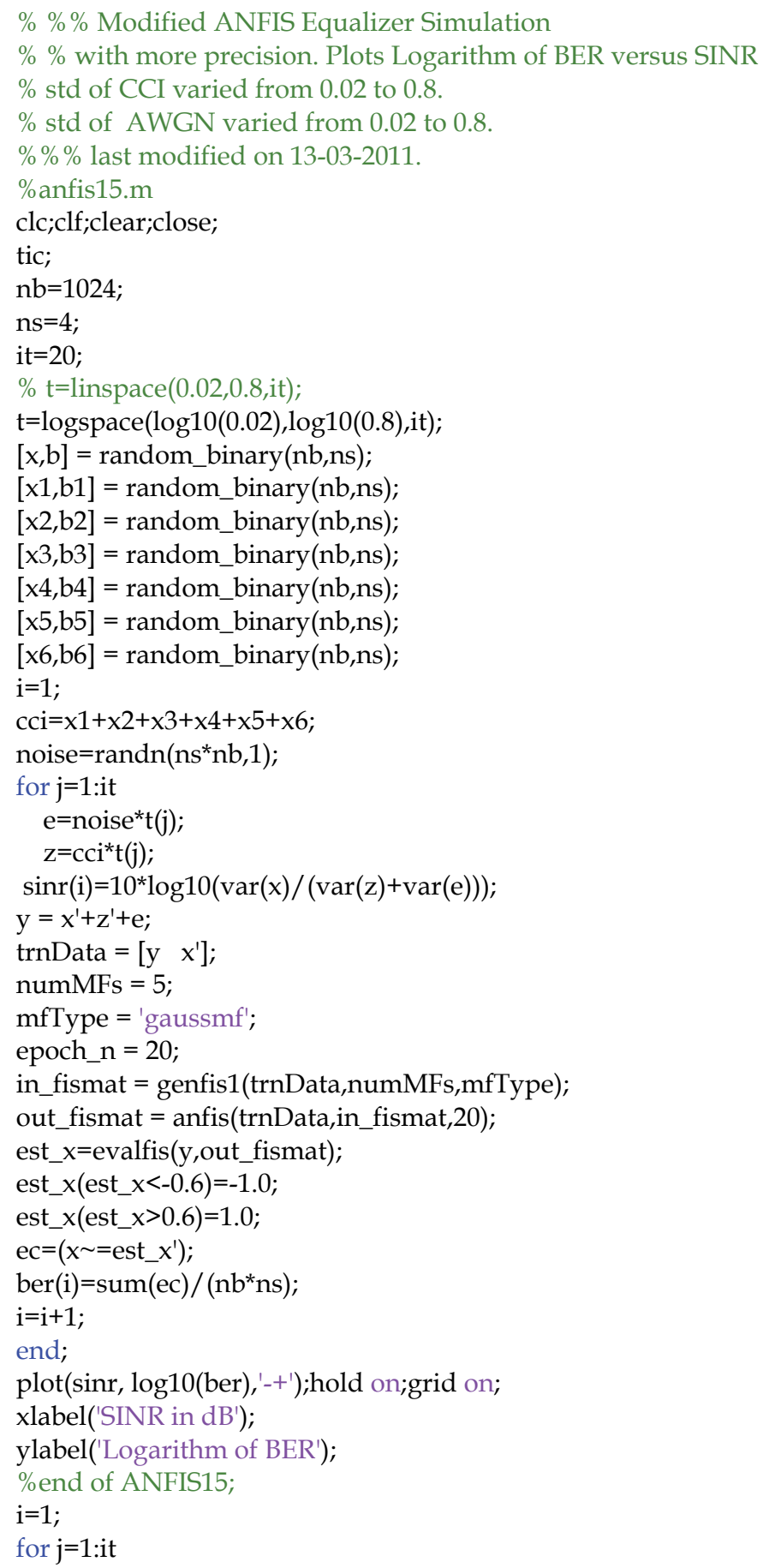




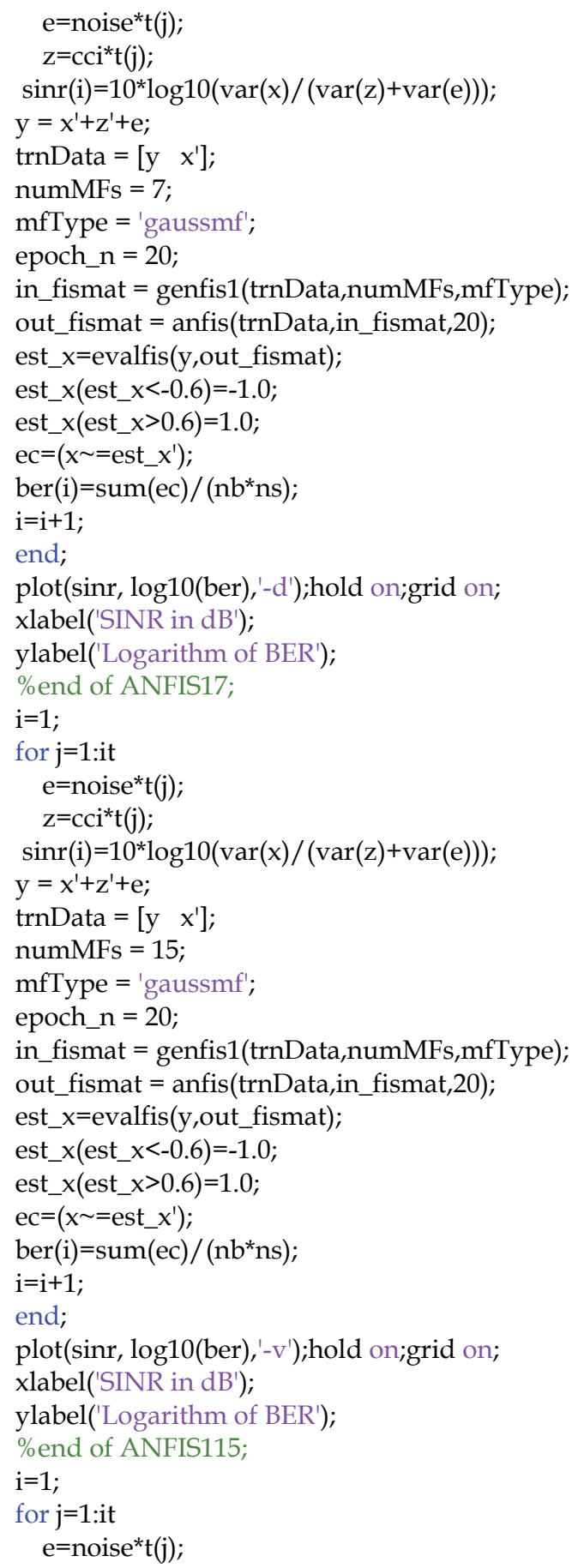




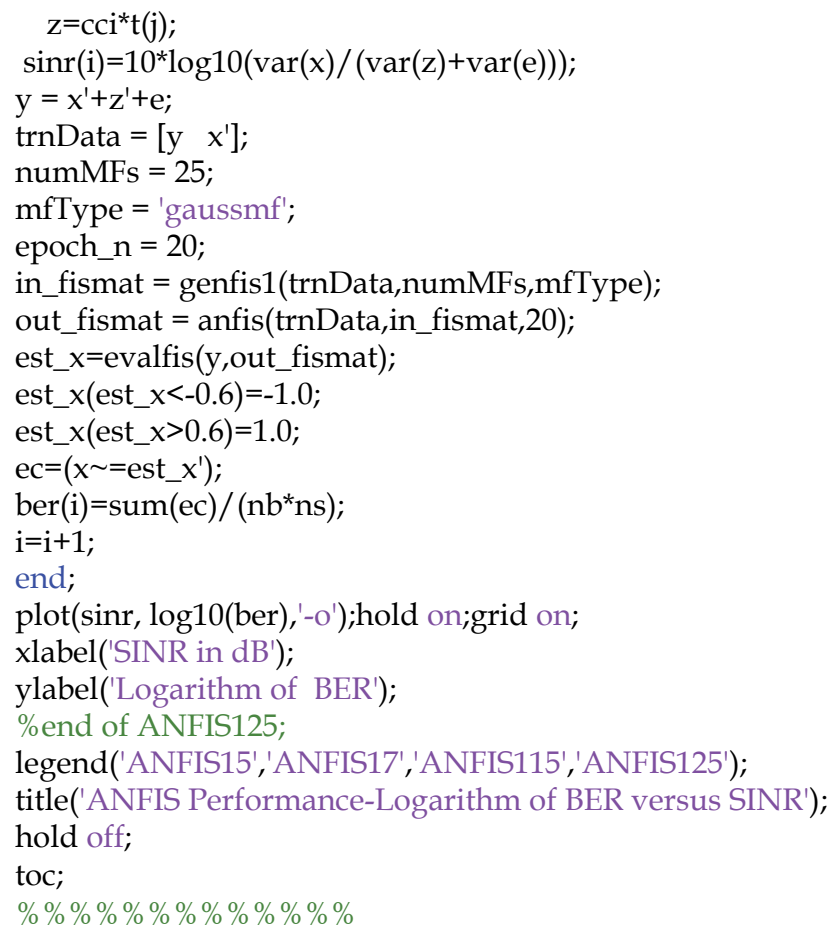

In another simulation, the $\log (B E R)$ at output of the equalizer is calculated for standard deviation of noise varying from 0.02 to 0.8 , and the for two versions of ANFIS Equalizers (ANFIS-115 and ANFIS-125) for 2048/4096 training data pairs and standard deviation of AWGN fixed at 0.42 , and the results are plotted in Figure 11. The MATLAB code used for the simulation is given below.

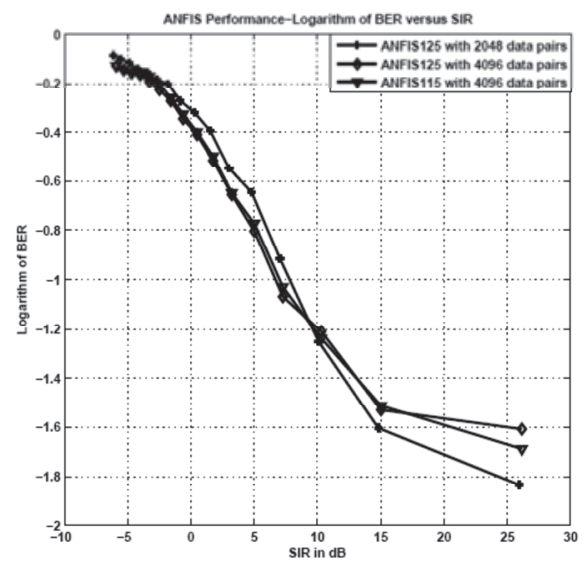

Fig. 11. Performance of ANFIS based Equalizer - Logarithm of BER at output versus SIR in dBs. 


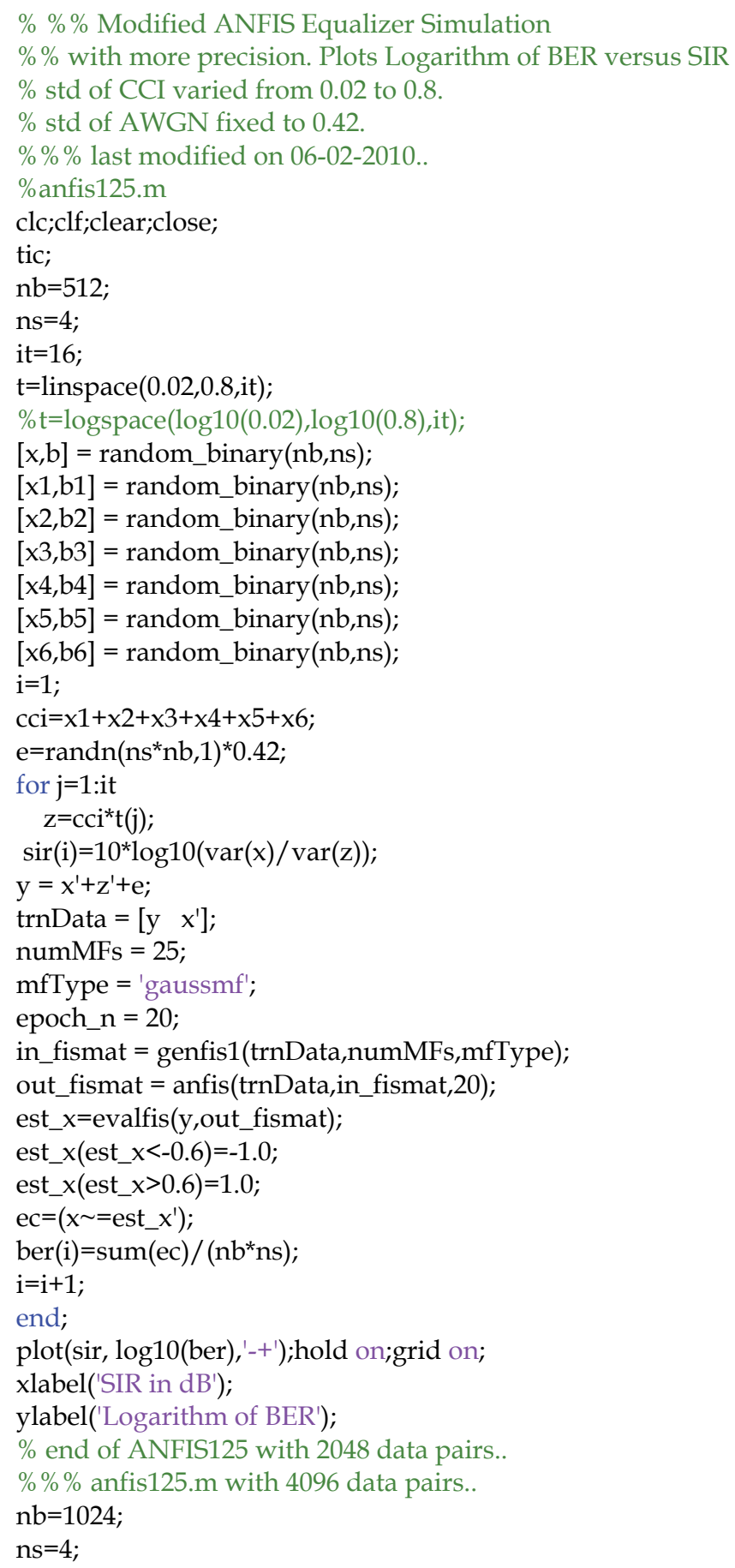


it $=16$;

$\mathrm{t}=$ linspace $(0.02,0.8, \mathrm{it})$;

$\% \mathrm{t}=\operatorname{logspace}(\log 10(0.02), \log 10(0.8), \mathrm{it})$;

$[\mathrm{x}, \mathrm{b}]=$ random_binary $(\mathrm{nb}, \mathrm{ns})$;

$[\mathrm{x} 1, \mathrm{~b} 1]=$ random_binary $(\mathrm{nb}, \mathrm{ns})$;

$[\mathrm{x} 2, \mathrm{~b} 2]=$ random_binary $(\mathrm{nb}, \mathrm{ns})$;

$[\mathrm{x} 3, \mathrm{~b} 3]=$ random_binary(nb,ns);

$[\mathrm{x} 4, \mathrm{~b} 4]=$ random_binary(nb,ns);

$[\mathrm{x} 5, \mathrm{~b} 5]=$ random_binary(nb,ns);

$[\mathrm{x} 6, \mathrm{~b} 6]=$ random_binary(nb,ns);

$\mathrm{i}=1$;

$\mathrm{cci}=\mathrm{x} 1+\mathrm{x} 2+\mathrm{x} 3+\mathrm{x} 4+\mathrm{x} 5+\mathrm{x} 6$;

$\mathrm{e}=\operatorname{randn}\left(\mathrm{ns}^{*} \mathrm{nb}, 1\right) * 0.42$;

for $j=1$ :it

$\mathrm{z}=\mathrm{CCi}^{*} \mathrm{t}(\mathrm{j})$;

$\operatorname{sir}(\mathrm{i})=10 * \log 10(\operatorname{var}(\mathrm{x}) / \operatorname{var}(\mathrm{z}))$;

$\mathrm{y}=\mathrm{x}^{\prime}+\mathrm{z}^{\prime}+\mathrm{e}$;

trnData $=\left[\begin{array}{ll}y & x^{\prime}\end{array}\right]$;

numMFs $=25$;

mfType = 'gaussmf';

epoch_n $=20$;

in_fismat = genfis1(trnData,numMFs,mfType);

out_fismat $=$ anfis(trnData,in_fismat,20);

est_x $=$ evalfis(y,out_fismat);

est_x $($ est_ $x<-0.6)=-1.0$;

est_x $($ est_x>0.6) $=1.0$;

$\mathrm{ec}=\left(\mathrm{x} \sim=\right.$ est_ $\left.\mathrm{x}^{\prime}\right)$;

$\operatorname{ber}(\mathrm{i})=\operatorname{sum}(\mathrm{ec}) /(\mathrm{ns} * \mathrm{nb})$;

$\mathrm{i}=\mathrm{i}+1$;

end;

plot(sir, log10(ber),'-d'); hold on; grid on;

$\%$ end of ANFIS125.m

$\% \%$ anfis115.m with 4096 data pairs..

$\mathrm{i}=1$;

$\mathrm{cci}=\mathrm{x} 1+\mathrm{x} 2+\mathrm{x} 3+\mathrm{x} 4+\mathrm{x} 5+\mathrm{x} 6$;

$\mathrm{e}=\operatorname{randn}\left(\mathrm{ns}^{*} \mathrm{nb}, 1\right) * 0.42$;

for $j=1$ :it

$\mathrm{z}=\mathrm{Cci}^{*} \mathrm{t}(\mathrm{j})$;

$\operatorname{sir}(\mathrm{i})=10 * \log 10(\operatorname{var}(\mathrm{x}) / \operatorname{var}(\mathrm{z}))$;

$\mathrm{y}=\mathrm{x}^{\prime}+\mathrm{z}^{\prime}+\mathrm{e}$;

$\operatorname{trnData}=\left[\mathrm{y} \mathrm{x}^{\prime}\right]$;

numMFs $=15 ; \%$ number of membership_rules

mf Type = 'gaussmf';

epoch_n $=20$;

in_fismat = genfis1 $($ trnData,numMFs,mfType);

out_fismat = anfis(trnData,in_fismat,20);

est_x=evalfis(y,out_fismat); 
est_x(est_x<-0.6)=-1.0;

est_x(est_x>0.6) $=1.0$;

$\mathrm{ec}=(\mathrm{x} \sim=$ est_ $\mathrm{x})$;

$\operatorname{ber}(\mathrm{i})=\operatorname{sum}(\mathrm{ec}) /\left(\mathrm{nb}^{*} \mathrm{~ns}\right)$;

$\mathrm{i}=\mathrm{i}+1$;

end;

plot(sir,log10(ber),'-v'); hold on;grid on;

$\%$ end of ANFIS115.m

legend('ANFIS125 with 2048 data pairs','ANFIS125 with 4096 data pairs','ANFIS115 with 4096 data pairs');

title('ANFIS Performance-Logarithm of BER versus SIR');

hold off;

toc;

$\% \% \% \% \% \% \% \%$

In this case, we can observe that the $\log (B E R)$ reduces as the $S I R$ in $d B$ increases, consistently. Also, when the number of training data pairs is increased, there is a marginal improvement in performance. The performance for the above ANFIS pairs, as regards $\log (B E R)$ at output of the equalizer versus SNR in $\mathrm{dBs}$ for standard deviation of co-channel interference signal fixed at 0.08 , is given in Figure 12 . The MATLAB code is also given.

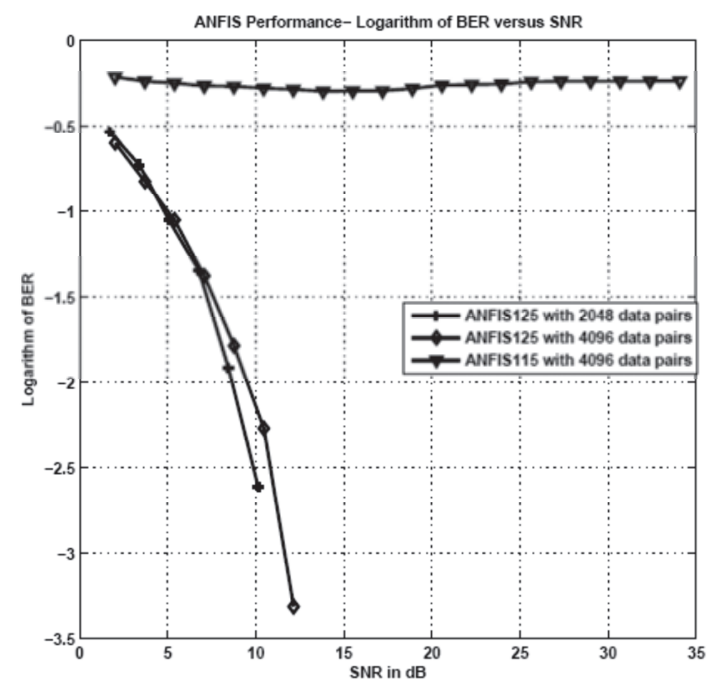

Fig. 12. Performance of ANFIS based Equalizer - Logarithm of BER versus SNR in dBs.

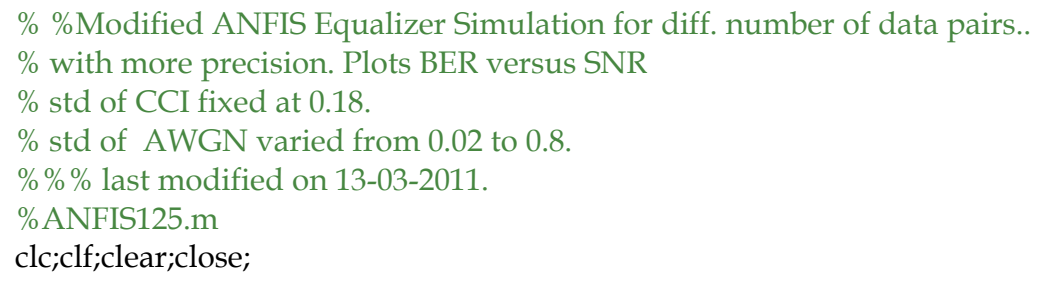


tic;

$\mathrm{nb}=512$;

ns $=4$;

it $=20$;

$\mathrm{t}=\log$ space $(\log 10(0.02), \log 10(0.8), \mathrm{it}) ; \% \%$

$[\mathrm{x}, \mathrm{b}]=$ random_binary(nb,ns);

$[\mathrm{x} 1, \mathrm{~b} 1]=$ random_binary $(\mathrm{nb}, \mathrm{ns})$;

$[\mathrm{x} 2, \mathrm{~b} 2]=$ random_binary $(\mathrm{nb}, \mathrm{ns})$;

$[\mathrm{x} 3, \mathrm{~b} 3]=$ random_binary $(\mathrm{nb}, \mathrm{ns})$;

$[\mathrm{x} 4, \mathrm{~b} 4]=$ random_binary(nb,ns);

$[\mathrm{x} 5, \mathrm{~b} 5]=$ random_binary(nb,ns);

$[\mathrm{x} 6, \mathrm{~b} 6]=$ random_binary(nb,ns);

$\mathrm{i}=1$;

cci $=x 1+x 2+x 3+x 4+x 5+x 6$;

noise $=\operatorname{randn}\left(\mathrm{ns}^{*} \mathrm{nb}, 1\right)$;

for $j=1$ :it

$\mathrm{e}=$ noise*t $\mathrm{t}$ );

$\mathrm{z}=\mathrm{cci}^{*} 0.08 ; \%$ std of cci is fixed as 0.08

$\operatorname{snr}(\mathrm{i})=10 * \log 10(\operatorname{var}(\mathrm{x}) / \operatorname{var}(\mathrm{e}))$;

$y=x^{\prime}+z^{\prime}+e$;

trnData $=\left[\begin{array}{ll}y & x^{\prime}\end{array}\right]$;

numMFs = 25;

mfType = 'gaussmf';

epoch_n $=20$;

in_fismat = genfis1(trnData,numMFs,mfType);

out_fismat $=$ anfis(trnData,in_fismat,20);

est_ $x=$ evalfis(y,out_fismat);

est_x $($ est_ $x<-0.6)=-1.0$;

est_x $($ est_x $>0.6)=1.0$;

$\mathrm{ec}=\left(\mathrm{x} \sim=\mathrm{est} \_\mathrm{x}\right)$;

$\operatorname{ber}(\mathrm{i})=\operatorname{sum}(\mathrm{ec}) /\left(\mathrm{nb}^{*} \mathrm{~ns}\right)$;

$\mathrm{i}=\mathrm{i}+1$;

end;

plot(snr, $\log 10$ (ber), '-+'); hold on;grid on;

xlabel('SNR in $\mathrm{dB}$ ');

ylabel('Logarithm of BER');

$\%$ end of ANFIS125;

$\mathrm{nb}=1024$;

$\mathrm{ns}=4$;

$\mathrm{t}=\log$ space $(\log 10(0.02), \log 10(0.8), \mathrm{it})$;

$[\mathrm{x}, \mathrm{b}]=$ random_binary $(\mathrm{nb}, \mathrm{ns})$;

$[\mathrm{x} 1, \mathrm{~b} 1]=$ random_binary $(\mathrm{nb}, \mathrm{ns})$;

$[\mathrm{x} 2, \mathrm{~b} 2]=$ random_binary(nb,ns);

$[\mathrm{x} 3, \mathrm{~b} 3]=$ random_binary $(\mathrm{nb}, \mathrm{ns})$;

$[\mathrm{x} 4, \mathrm{~b} 4]=$ random_binary $(\mathrm{nb}, \mathrm{ns})$;

$[\mathrm{x} 5, \mathrm{~b} 5]=$ random_binary(nb,ns); 
$[\mathrm{x} 6, \mathrm{~b} 6]=$ random_binary(nb,ns);

$\mathrm{i}=1$;

$\mathrm{cci}=\mathrm{x} 1+\mathrm{x} 2+\mathrm{x} 3+\mathrm{x} 4+\mathrm{x} 5+\mathrm{x} 6$;

noise $=$ randn $\left(n s^{*} \mathrm{nb}, 1\right)$;

for $j=1$ :it

$\mathrm{e}=$ noise ${ }^{*} \mathrm{t}(\mathrm{j})$;

$\mathrm{z}=\mathrm{cci}^{*} 0.08 ; \%$ std of cci is fixed as 0.18

$\operatorname{snr}(\mathrm{i})=10 * \log 10(\operatorname{var}(\mathrm{x}) / \operatorname{var}(\mathrm{e}))$;

$\mathrm{y}=\mathrm{x}^{\prime}+\mathrm{z}^{\prime}+\mathrm{e}$;

trnData $=\left[\begin{array}{ll}y & x^{\prime}\end{array}\right]$;

numMFs = 25;

mfType = 'gaussmf';

epoch_n $=20$;

in_fismat = genfis1(trnData,numMFs, mfType);

out_fismat = anfis(trnData,in_fismat,20);

est_x=evalfis(y,out_fismat);

est_x $($ est_x $<-0.6)=-1.0$;

est_x (est_ $x>0.6)=1.0$;

$\mathrm{ec}=\left(\mathrm{x} \sim=\right.$ est_ $\left.\mathrm{x}^{\prime}\right)$;

$\operatorname{ber}(\mathrm{i})=\operatorname{sum}(\mathrm{ec}) /\left(\mathrm{nb}^{*} \mathrm{~ns}\right)$;

$\mathrm{i}=\mathrm{i}+1$;

end;

plot(snr, log10(ber),'-d');hold on;grid on;

xlabel('SNR in $\mathrm{dB}$ ');

ylabel('Logarithm of BER');

$\%$ end of ANFIS125;

$\% \%$ anfis $115 . m$

$\mathrm{t}=\log$ space $(\log 10(0.02), \log 10(0.8), \mathrm{it})$;

$[\mathrm{x}, \mathrm{b}]=$ random_binary $(\mathrm{nb}, \mathrm{ns})$;

$[\mathrm{x} 1, \mathrm{~b} 1]=$ random_binary(nb,ns);

$[\mathrm{x} 2, \mathrm{~b} 2]=$ random_binary(nb,ns);

$[\mathrm{x} 3, \mathrm{~b} 3]=$ random_binary $(\mathrm{nb}, \mathrm{ns})$;

$[\mathrm{x} 4, \mathrm{~b} 4]=$ random_binary $(\mathrm{nb}, \mathrm{ns})$;

$[\mathrm{x} 5, \mathrm{~b} 5]=$ random_binary $(\mathrm{nb}, \mathrm{ns})$;

$[\mathrm{x} 6, \mathrm{~b} 6]=$ random_binary $(\mathrm{nb}, \mathrm{ns})$;

$\mathrm{i}=1$;

for $j=1$ :it

$\mathrm{e}=$ noise* $\mathrm{t}(\mathrm{j})$;

$\mathrm{z}=\mathrm{CCi}^{*} .08$;

$\operatorname{snr}(\mathrm{i})=10 * \log 10(\operatorname{var}(\mathrm{x}) / \operatorname{var}(\mathrm{e}))$;

$\mathrm{y} 1=\mathrm{x}^{\prime}+\mathrm{z}^{\prime}+\mathrm{e}$;

trnData $=\left[y 1 x^{\prime}\right]$;

numMFs $=15 ; \%$ number of membership_rules

mfType = 'gaussmf';

epoch_n $=20$;

in_fismat = genfis1(trnData,numMFs, $m$ fType); 


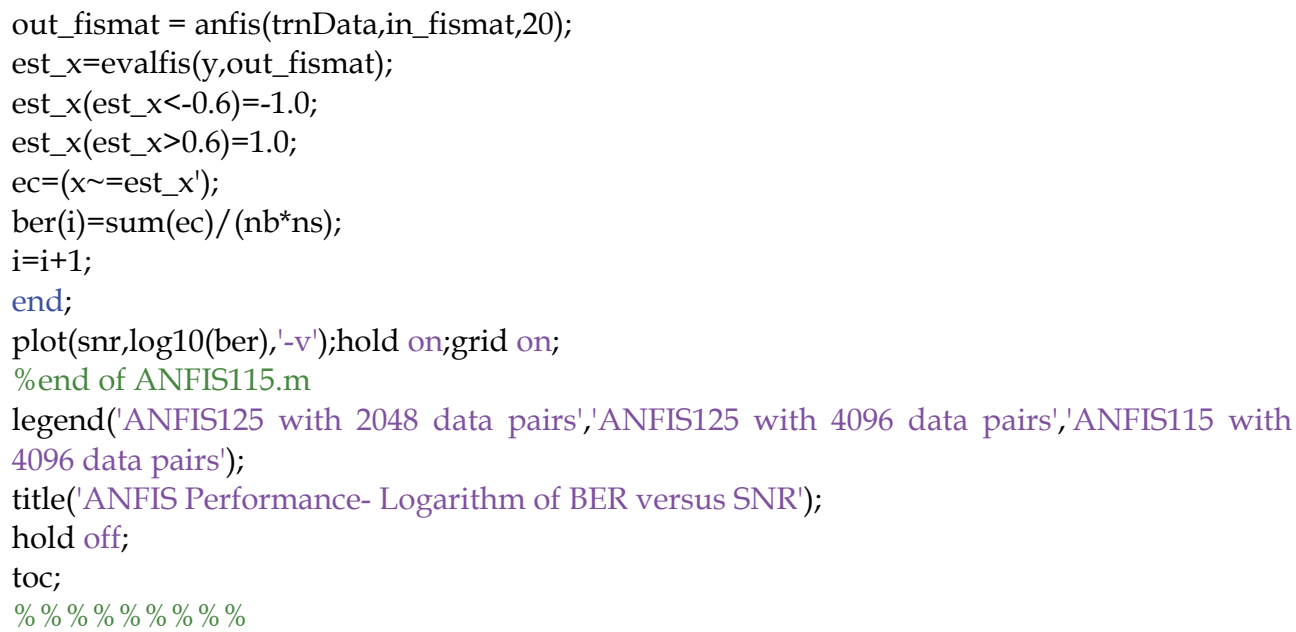

Note that in this case, ANFIS-115 fails to converge and hence there is no variation in performance even when the SNR is as high as $35 \mathrm{~dB}$. Again, the performance is marginally better, when the number of training data pairs is increased. A plot of the performance of two different ANFIS structures (average BER at the output of the equalizer versus SNR and standard deviation of BER at the output of the equalizer versus standard deviation of AWGN) based on 100 Monte-Carlo (MC) simulations is given in Figure 12 for ANFIS-115 and ANFIS-125 structures. 4096 training data pairs are used in the simulation. The Mean(BER) versus SNR performance improves consistently, as the SNR increases. The $\operatorname{std}(B E R)$ versus $S N R$ performance, on the otherhand, deteriorates as the $\operatorname{std}(A W G N)$ increases, consistently. Performances are marginally better for ANFIS-125 based equalizer. We will now consider the interpretation of the results of simulation of various ANFIS based equalizers.

\subsection{Interpretation of results of simulations of ANFIS based equalizers}

The following observations are made based on Figures 9, 10, 11, 12 and 13 and Tables 1 as well as results of simulations with less number of data pairs:

- With more number of training data pairs, BER at the output of the equalizer is reduced. This is due to the fact that the ANFIS gets optimally tuned with more training data pairs.

- As shown in Figure 10, performance of all ANFIS Equalizers w.r.t. $\log (B E R)$ at the output of the equalizer versus SINR, is nearly identical. When the SINR is above $-10 d B$, practically the $\log (B E R)$ becomes close to zero. However, ANFIS-125 performs slightly better than other structures.

- $\quad$ The performance of ANFIS-125 w.r.t. $\log (B E R)$ at the output of the equalizer versus SIR is almost identical with 2048 or 4096 data pairs. However for ANFIS-115, performance is slightly poor.

- As it is shown in Figure 12, the performance of ANFIS-125 w.r.t. $\log (B E R)$ at the output of the equalizer versus SNR is almost identical with 2048 or 4096. data pairs. However for ANFIS-115, performance is very poor even at a SNR of $35 \mathrm{~dB}$. This is due to the fact 
that equalizer model with ANFIS structure fails to perform when the number of rules is 15. The AWGN overwhelms the signal, when number of rules for the ANFIS is 15 or less.

- For MISO or MIMO systems, increasing the number of membership functions is the option for accurate system modeling, since in these cases number of inputs applied to the ANFIS is two or more, and hence it will not be optimal to increase the number of internal inputs in ANFIS.

- An optimal ANFIS structure can be obtained based on the training time and the maximum error that can be tolerated. As indicated in Figure 13, at higher values of standard deviation of AWGN, and that of standard deviation of BER will be less with more number of membership functions. Hence standard deviation of BER at the output of the equalizer can be yet another criterion in selecting a particular ANFIS structure.

\section{The equalization of Ultra-Wide Band channels using ANFIS}

The Ultra-Wide Band (UWB) is an emerging wireless technology that has recently gained much interest from the communication research and industry (Molisch, 2005a). UWB systems possess unique characteristics and capabilities that make them suitable for short range, high-speed wireless communications (Molisch, 2005b). The UWB systems use signals that are based on repetitive transmissions of short pulses formed by using a single basic pulse shape. The transmitted signals have an extremely low power spectral density and occupy very large bandwidth of several GHz. Thus the UWB systems can operate with negligible interference to the existing radio systems. UWB can provide very high bit rate, low-cost, low-power wireless communication for wide variety of systems: personal computer, TV, VCR, CD, DVD, and MP3 players (Algans et.al., 2002).
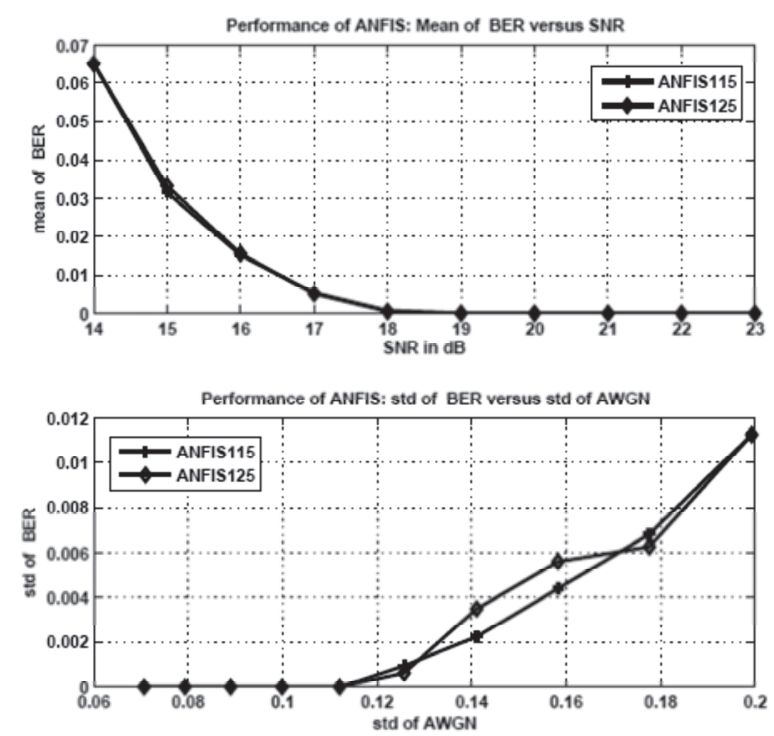

Fig. 13. Performance of ANFIS Equalizers: (a) Mean BER at out of the equalizer versus SNR in dBs, and (b) Standard Deviation of BER at output of the equalizer versus Standard Deviation of AWGN. 
As per FCC recommendations, UWB systems have the following characteristics:

- They have a relative bandwidth that is larger than $25 \%$ of the carrier frequency and/or an absolute band-width more than $500 \mathrm{MHz}$.

- They occupy a frequency band of $3.1 \mathrm{GHz}$ to $10.6 \mathrm{GHz}$.

- FCC have recently allocated $7.5 \mathrm{GHz}$ of spectrum for unlicensed commercial UWB communication systems.

- Maximum radiated power is $75 \mathrm{nW} / \mathrm{MHz}(-41.32 \mathrm{dBm} / \mathrm{MHz})$ (Molisch, 2005a).

The following are the significant merits of UWB:

1. Accurate position location and ranging, due to the better time resolution.

2. No significant multipath fading due to better time resolution.

3. Multiple access due to wide transmission bandwidths.

4. Possibility of extremely high data rates.

5. Covert communications due to low transmission power operation.

6. Possible easier material penetration due to the presence of components at different frequencies.

\subsection{The channel covariance matrix formulation for UWB channels}

The Shadow-fading fluctuations of the average received power are known to be lognormally distributed. Recently, for macrocell scenarios, the fluctuations in delay and angle spread are shown to behave similarly (Algans et.al., 2002). The reason is that these quantities are sums of powers of individual sub-paths times the square of their corresponding delay times or angles. Since the powers are log-normally distributed and sums of lognormal variables are approximately) log-normal, this implies that angle-spreads and delay-spreads have log-normal distributions (Beaulieu et.al., 1995). This motivation of how angle spread and delay spread are log-normally distributed also suggests that they will be correlated with shadow fading and each other. Let us assume that $X_{1 n}, X_{2 n}, X_{3 n}, \ldots$ are zero-mean, unit-variance Gaussian random variables, representing the signals received at base station $n$. Then we define:

$$
\begin{gathered}
\rho_{D A}=E\left[X_{1 n}, X_{2 n}\right] \\
\rho_{D F}=E\left[X_{2 n}, X_{3 n}\right] \\
\rho_{A F}=E\left[X_{3 n}, X_{1 n}\right] \\
\zeta=E\left[X_{3 n}, X_{3 m}\right]
\end{gathered}
$$

In particular, $\sigma_{S F, n}$ (variance of shadow fading component w.r.t. to base station, $n$ ) is negatively correlated with $\sigma_{D S, n}$ (variance of delay spread) and $\sigma_{A S, n}$ (variance of angle spread), while the latter two have positive correlations with each other. It should be noted that this relationship does not hold for the angle-spread at the mobile since the different paths with distinct angles do not necessarily lead to such pronounced differences in the delays. These correlations can be expressed in terms of a covariance matrix $\mathrm{A}$, whose $A_{i j}$ component represents the correlations between $X_{i n}$ and $X_{j n}$, with $i, j=1,2,3$. Note that the matrix $A$ is symmetrical. Measurements of cross-correlations of these parameters between different base stations are more difficult. In particular, only correlations between shadow-fading components have been adopted. These correlations are assumed to be the same between any two different base-stations and are denoted by $\zeta$. For simplicity and due to lack of further data, the cross-correlation matrix between the $X_{\text {in }}$ triplet $(i=1,2,3)$ of different base-stations are assumed to be given by the following matrix $B$. 


$$
\mathrm{A}=\left[\begin{array}{ccc}
1 & \rho D A & \rho D F \\
\rho D A & 1 & \rho A F \\
\rho D F & \rho A F & 1
\end{array}\right], \text { and } \mathrm{B}=\left[\begin{array}{ccc}
0 & 0 & 0 \\
0 & 0 & 0 \\
0 & 0 & \zeta
\end{array}\right]
$$

\subsection{Simulation of ANFIS equalizer for UWB channels}

We will now consider the simulation setup for UWB channels based on the Channel Covariance Matrices (CCM). The following extended channel covariance matrix was used in the simulations:

$$
\mathrm{A}=\left[\begin{array}{cccc}
1 & 0.8 & -0.7 & 0.6 \\
0.8 & 1 & -0.6 & 0.5 \\
-0.7 & -0.6 & 1 & 0.5 \\
0.6 & 0.5 & 0.5 & 1
\end{array}\right] \text {, and } A^{\prime}=\left[\begin{array}{cccc}
1+\alpha & 0.8+\beta & -0.7+\gamma & 0.6+\delta \\
0.8+\beta & 1+\alpha & -0.6+\varepsilon & 0.5+\eta \\
-0.7+\gamma & -0.6+\varepsilon & 1+\alpha & 0.5+\zeta \\
0.6+\delta & 0.5+\eta & 0.5+\zeta & 1+\alpha
\end{array}\right]
$$

$A^{\prime}$ indicate the modified CCM corrupted by CCI and AWGN. We use an ANFIS with the following parameters in the equalizer:

- One-input One-output ANFIS.

- 20 Rules.

- Gaussian membership functions.

- Maximum spread in CCM parameters is 0.5 ([0.0:0.1:0.5]).

The structure of the ANFIS is given in Figure 14. The simulation results are given in Figure 15. The MATLAB code for the simulation is appended below.

$\%$ Program to model the wideband channel

$\%$ Using the covariance matrix.

\% Last modified on 13-03-2011.

clc; clf; clear all;

tic;

covm $=[1.8-.7 .6 ; .81-.6 .5$;

$-.7-.61 .5 ; .6 .5 .51]$

for $\mathrm{mc}=1: 4 \%$ for $4 \mathrm{MC}$ simulations.

mxerr=[];

for spr=.1:.01:.5\% for .. values of spread.

$\mathrm{x}=\operatorname{covm}(:)$;

$y(1)=x(1)+$ randn + spr; $\%$ first element with spread..

$y(2)=x(2)+r a n d n+s p r ; \%$ second element with spread..

$y(3)=x(3)+$ randn+spr; \% third element with spread..

$\mathrm{y}(4)=x(4)+$ randn+spr; $\%$ fourth element with spread. .

$\mathrm{y}(5)=x(2) ; \mathrm{y}(6)=x(1) ; \mathrm{y}(11)=x(1) ; \mathrm{y}(16)=x(1)$;

$y(7)=x(7)+$ randn+spr;

$\mathrm{y}(8)=x(8)+$ randn+spr;

$\mathrm{y}(9)=\mathrm{x}(3)$;

$y(10)=x(10)+$ randn + spr;

$y(12)=x(12)+$ randn + spr;

$\mathrm{y}(13)=x(4) ; y(14)=x(12) ; y(15)=x(12)$;

$\mathrm{y}=\mathrm{y}(1: 16)$;

trnData $=\left[\begin{array}{ll}y^{\prime} & x\end{array}\right]$;

numMFs $=20 ; \%$ No of Membership functions.. 

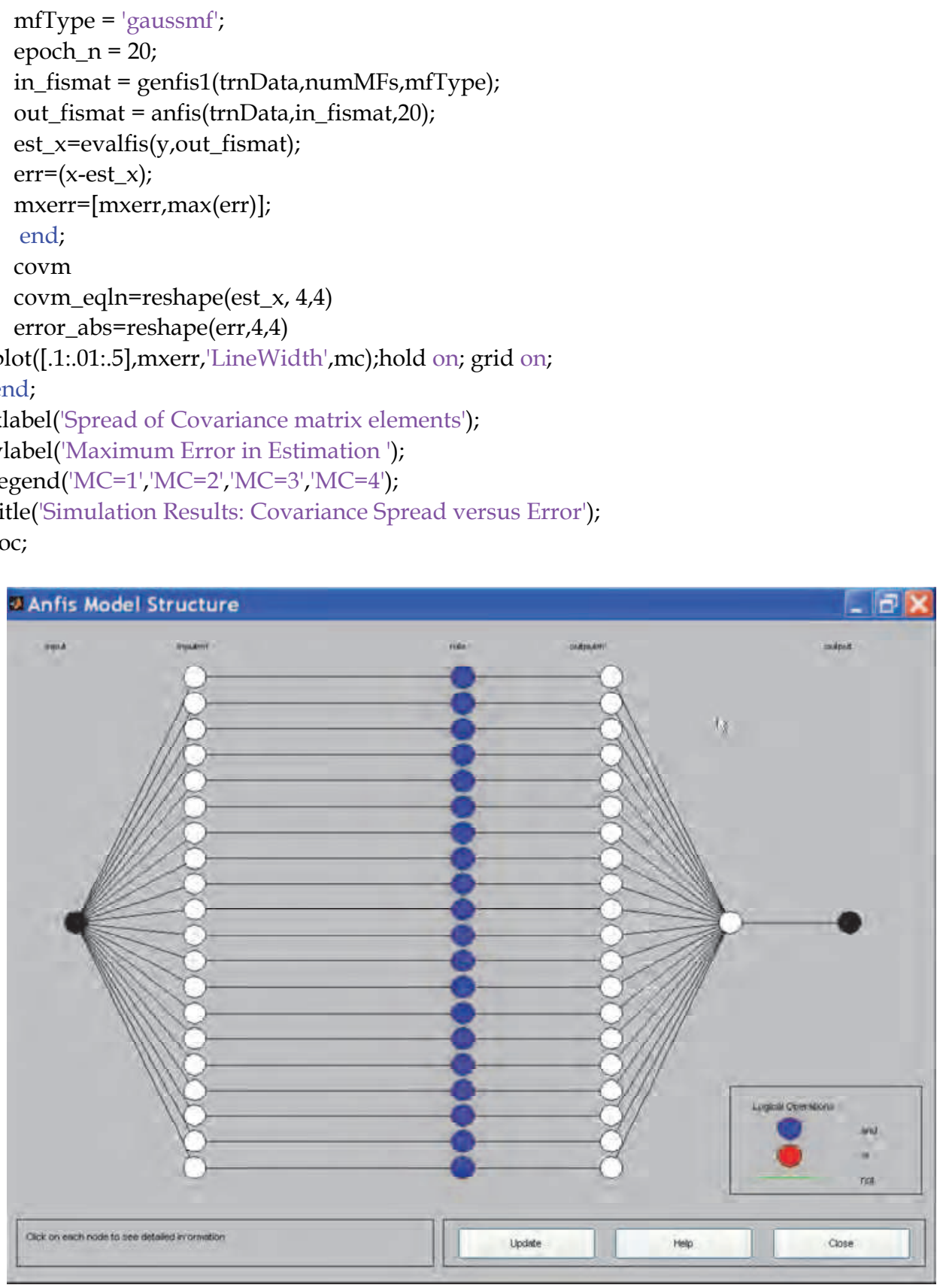

Fig. 14. ANFIS Model Structure Used for UWB Channel Equalization: Number of Inputs=1, Number of Outputs=1, Number of Rules=20, and Type of Membership Function- Gaussian. 


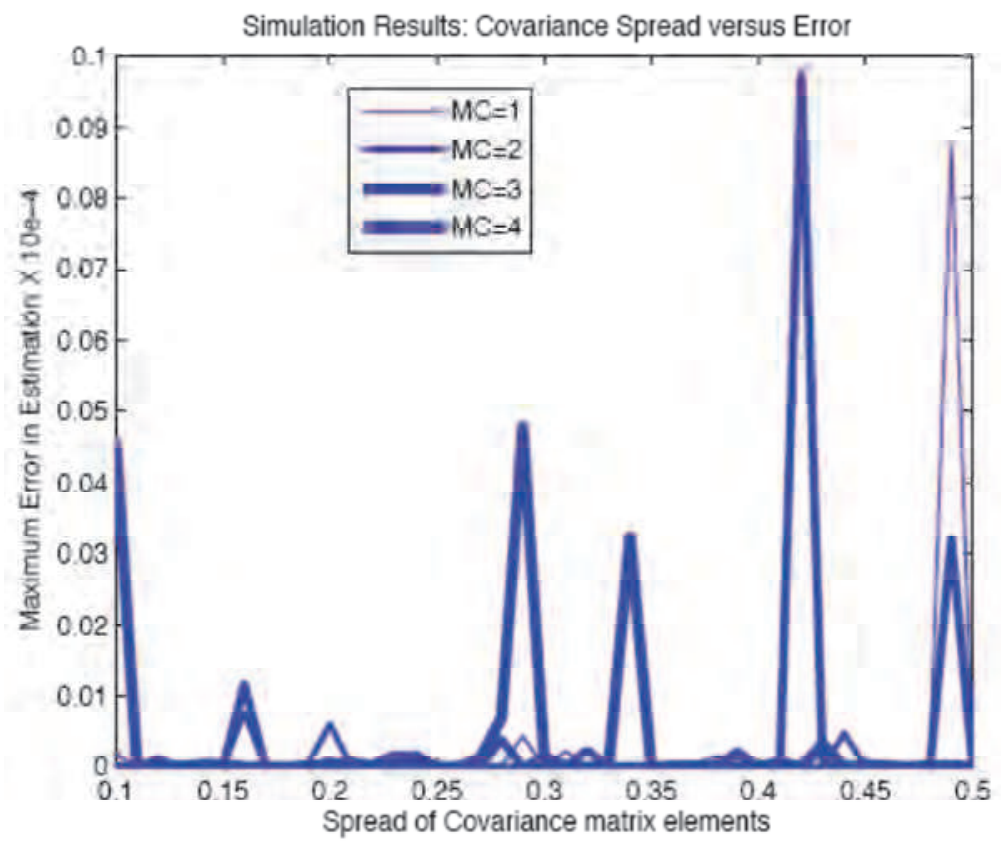

Fig. 15. Results of Simulation - ANFIS based Equalizer for UWB Channels.

It shows that the ANFIS model is capable of estimating the CCM parameters with almost negligible error.

\section{Concluding remarks on ANFIS equalizers}

In this chapter, we considered an alternative solution to the non-linear channel equalization problem. Several ANFIS equalizer structures are considered, with varying number of inputs and membership functions. It was found that the BER versus SINR performances of all of them were almost the same. However, at low values of SNR, ANFIS structure with more number of nodes (or more number of rules) performed slightly better. But as the number of nodes in the ANFIS structure was increased, convergence time was also increased. The number of nodes in the ANFIS structure is a function of the number of inputs, membership functions and outputs. The time for convergence increases, as the number of inputs or membership functions increases.

It is also shown that equalizers based on ANFIS can be suitably adapted for UWB channels as well. A channel co-variance matrix (CCM) formulation was used to model the UWB channel. It was shown that the estimate of the CCM was better when the spread in parameters was small.

\section{Acknowledgements}

The author is most thankful to Prof M Harisankar, Adjunct Professor, Department of IT, Indian Institute of Management, Kozhikode for his valuable guidance, mentoring and technical support while authoring the chapter. 


\section{References}

B.Widrow et.al. (1975). The Complex LMS Algorithm, Proceedings of the IEEE, Vol.63, pp.719-720, April.

John G.Proakis and Masoud Salehi, (2002). Communication Systems Engineering, 2nd edition, ISBN 978-0130617934, Pearson Education.

Y. Okumura, E. Ohmori, T. Kawano, and K. Fukua, (1968). Field Strength and its Variability in UHF and VHF Land-mobile Radio Service, Rev. Elec. Commun. Lab., Vol. 16, No. 9.

M. Hata, (1980). Empirical Formula for Propagation Loss in and Mobile Radio Services, IEEE Transactions on Vehicular Technology, Vol. 29, pp. 317-325, August.

M.S. Smith, J.E.J. Dalley, (2000). A New Methodology for Deriving Pathloss Models from Cellular Drive Test Data, Proceedings of AP2000 Conference, Davos, Switzerland, April.

Cheng Jian Lin and Wen Hao Ho, (2004). Blind Equalization Using Pseudo-Gaussian-Based Compensatory Neuro-Fuzzy Filters, International Journal of Applied Science and Engineering, Vol.2, No.1, pp.72-89, January.

Li-Xin Wang and Jerry M. Mendel, (1993). An RLS Fuzzy Adaptive Filter, with Application to Nonlinear Channel Equalization, IEEE Transactions on Fuzzy Systems, Vol.1, pp.895-900, August.

Qilian Liang and Jerry M. Mendel, (2000). Overcoming Time-Varying Co-Channel Interference Using Type-2 Fuzzy Adaptive Filters, IEEE Transactions on Circuits and Systems-II:Analog and Digital Signal Processing, Vol.47,No.12, pp.1419-1428, December.

Jyh-Shing Roger Jang, (1993). ANFIS: Adaptive-Network-Based Fuzzy Inference System, IEEE Transactions on Systems, Man, and Cybernetics, Vol.23, No.3, pp.665-685, May/June.

Andreas F. Molisch, (2005a). Ultra-wideband Propagation Channels-Theory, Measurement, and Modeling, IEEE Transactions on Vehicular Technology, Vol. 54, No. 5, pp. 1528-1545, September.

Andreas F. Molisch, (2005b). Wireless Communications, ISBN 81-265-1056-0, John Wiley.

A. Algans, K. I. Pedersen, and P. E. Mogensen, (2002). Experimental Analysis of the Joint Statistical Properties of Azimuth Spread, Delay Spread, and Shadow Fading, IEEE Journal on Selected Areas in Communication, Vol. 20, No. 3, pp. 523-531, April.

N. C. Beaulieu, A. A. Abu-Dayya, and P. J. McLane, (1995). Estimating the Distribution of a Sum of Independent Lognormal Random Variables, IEEE Transactions on Communications, Vol. 43, No. 12, pp. 2869-2873, December. 


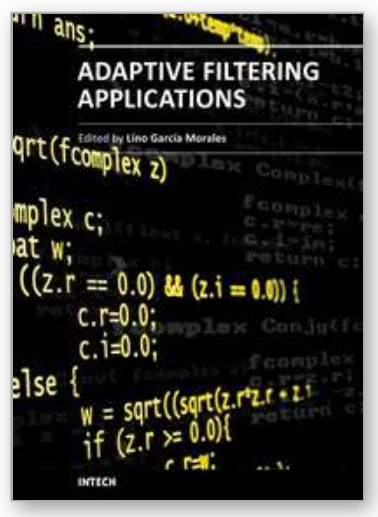

\author{
Adaptive Filtering Applications \\ Edited by Dr Lino Garcia
}

ISBN 978-953-307-306-4

Hard cover, 400 pages

Publisher InTech

Published online 24, June, 2011

Published in print edition June, 2011

Adaptive filtering is useful in any application where the signals or the modeled system vary over time. The configuration of the system and, in particular, the position where the adaptive processor is placed generate different areas or application fields such as: prediction, system identification and modeling, equalization, cancellation of interference, etc. which are very important in many disciplines such as control systems, communications, signal processing, acoustics, voice, sound and image, etc. The book consists of noise and echo cancellation, medical applications, communications systems and others hardly joined by their heterogeneity. Each application is a case study with rigor that shows weakness/strength of the method used, assesses its suitability and suggests new forms and areas of use. The problems are becoming increasingly complex and applications must be adapted to solve them. The adaptive filters have proven to be useful in these environments of multiple input/output, variant-time behaviors, and long and complex transfer functions effectively, but fundamentally they still have to evolve. This book is a demonstration of this and a small illustration of everything that is to come.

\title{
How to reference
}

In order to correctly reference this scholarly work, feel free to copy and paste the following:

K. C. Raveendranathan (2011). An Introduction to ANFIS Based Channel Equalizers for Mobile Cellular Channels, Adaptive Filtering Applications, Dr Lino Garcia (Ed.), ISBN: 978-953-307-306-4, InTech, Available from: http://www.intechopen.com/books/adaptive-filtering-applications/an-introduction-to-anfis-based-channelequalizers-for-mobile-cellular-channels

\section{INTECH}

open science | open minds

\section{InTech Europe}

University Campus STeP Ri

Slavka Krautzeka 83/A

51000 Rijeka, Croatia

Phone: +385 (51) 770447

Fax: +385 (51) 686166

www.intechopen.com

\section{InTech China}

Unit 405, Office Block, Hotel Equatorial Shanghai

No.65, Yan An Road (West), Shanghai, 200040, China

中国上海市延安西路65号上海国际贵都大饭店办公楼405单元

Phone: +86-21-62489820

Fax: +86-21-62489821 
(C) 2011 The Author(s). Licensee IntechOpen. This chapter is distributed under the terms of the Creative Commons Attribution-NonCommercialShareAlike-3.0 License, which permits use, distribution and reproduction for non-commercial purposes, provided the original is properly cited and derivative works building on this content are distributed under the same license. 